AperTO - Archivio Istituzionale Open Access dell'Università di Torino

\title{
Generalizing Montague's Theorem on Recursive Definitions
}

\section{This is the author's manuscript}

Original Citation:

Availability:

This version is available http://hdl.handle.net/2318/1812513

since 2022-01-26T14:48:51Z

Published version:

DOI:10.1215/00294527-2021-0027

Terms of use:

Open Access

Anyone can freely access the full text of works made available as "Open Access". Works made available under a Creative Commons license can be used according to the terms and conditions of said license. Use of all other works requires consent of the right holder (author or publisher) if not exempted from copyright protection by the applicable law. 
Submitted on July 17, 2019 to the Notre Dame Journal of Formal Logic

Volume ??, Number??,

\title{
Generalising Montague's Theorem on Recursive Definitions
}

\author{
Edoardo Rivello
}

\begin{abstract}
I study several kinds of generalisations of Montague's theorem on the method of definition of a function by recursion on a well-founded relation. In particular, I extend Montague's theorem to cover the following situations: recursion by a valuation system, non-well-founded recursion, and recursion on a dependence operator. By means of these extensions, several constructions employed in formal theories of truth and paradox can be recast as special applications of the generalised version of Montague's theorem.
\end{abstract}

\section{Introduction}

The principle of definition of a function by recursion on a well-founded relation can be stated as follows. Let $A$ be any set and let ${ }^{[A]} B$ denote the set of all partial functions from $A$ to some set $B$. Let $G: A \times{ }^{[A]} B \rightarrow B$ be any function and let $R \subseteq A \times A$ be any binary relation:

Fact 1.1 (Montague [6]) If $R$ is well-founded on $A$ then there exists one and only one function $f: A \rightarrow B$ such that

$$
f(x)=G\left(x, f\left\lceil x^{R}\right),\right.
$$

holds for every $x \in A$, where $x^{R}=\{y \in A \mid y R x\}$.

We will refer to Fact 1.1 as to "Montague's theorem". Montague's theorem is a result in standard set theory ${ }^{1}$ which generalises the usual mathematical principle of definition by recursion on the natural numbers, leading to a method of definition which proves to be a powerful tool in every field where set-theoretic techniques can be applied.

However, sometimes we find ourselves in situations in which a straightforward application of Montague's theorem is not available, yet we feel that a "recursive definition" of some function should be the natural one. The case roughly presents

2010 Mathematics Subject Classification: Primary 03E47; Secondary 03E75, 03A10

Keywords: Recursive definition, well-foundedness, dependence, truth 
as following. The recursive equation (1) conveys the intuitive idea that "the value of the function $f$ at $x$ is determined by the values $f$ itself assigns to the elements of the set on which $x$ depends on". When we are trying to define a function, it can happen that we have in mind some sort of intuitive recursive clause which suggests an application of Montague's theorem. However, in formalising the recursive clause as a recursive equation like (1), we may possibly find ourselves in one or more of the following cases: (a) the value of $f$ at $x$ is not determined by the application of a function like $G$, rather by some other kind of "valuation"; (b) the dependence of $x$ from other elements of $A$ is not represented by a binary relation like $R$, but by some other device; (c) $x$ depends on itself or, in any case, its dependency exhibits some form of (apparently unavoidable) circularity.

Our goal is to generalise Montague's theorem in such a way some situations, falling under the informal picture given above, could become tractable.

In particular, we will investigate the following cases: (a) a valuation system on $A$, roughly an operator on partial functions, replaces the function $G$ in the role of evaluating the value of $f$ at $x$ given some others values of $f$; (b) a monotone operator on $A$ replaces the binary relation $R$ in representing the notion of dependence and, accordingly, the notion of groundedness plays the role of well-foundedness; (c) the notion of intrinsicity replaces well-foundedness as a criterion ensuring existence and uniqueness of a "large" recursive function also defined on some elements of $A$ which are "circular" with respect to the given notion of dependence.

The motivation and driving ideas for this kind of generalisation come from the field of formal theories of truth and paradox. In those works we encounter mathematical constructions of the extension of the truth predicate that formalise a sort of intuitive "recursion" along a relation of semantic dependence. Due to inherent circularities in the semantics of truth (the semantic value of a sentence of the form $\operatorname{Tr}\ulcorner\phi\urcorner$ intuitively depends on the semantic value of the sentence $\phi$ which, in turn, could contain instances of the truth predicate $\operatorname{Tr}, \ldots$ ) usually the dependence relation is non-well-founded; in some cases it cannot not even be represented by a simple binary relationship between sentences. For these reasons, the form of recursion captured by Montague's theorem is not immediately recognisable in those mathematical constructions and the theorem itself is not directly applicable.

The purpose of this article is to abstract from several ad hoc forms of "recursion", used in formal theories of truth, a common pattern and to encode it in a generalisation of Montague's theorem which can be usefully applied to this and other similar situations.

In the remaining part of this Introduction we will fix the mathematical setting and provide some useful lemmata on the abstract notions of intrinsicity and determinacy that will constitute the main components of the subsequent treatment of recursion. Section 2 is devoted to (a) give precise definitions of the notions involved in stating and in generalising Montague's theorem, and (b) formulate and prove the main result, Theorem 2.15, that provides a "simultaneous" generalisation of Montague's theorem in all three directions sketched above. Examples of application of this generalised form of recursion, taken from the field of formal theories of truth, are provided in the subsequent sections, both for illustrating and for motivating the generalisations achieved. Section 3 focuses on the special case of recursion on a binary relation: on one hand, this choice allows us to emphasise the role of valuation systems and non-well-founded recursion; on the other hand, whenever dependence is represented 
by a binary relation, we can prove that recursion can be characterised in terms of the fixed points of a suitably defined monotone operator. Finally, Section 4 investigates the special case in which the notion of dependence is represented by an "internal" dependence operator, namely, by a monotone operator defined in terms of the valuation system. In this case, we find conditions for (a) performing a definition by recursion in the generalised sense, and (b) characterising the "grounded" part of the defined function by means of a definition by transfinite recursion.

General mathematical preliminaries Throughout the paper "iff" abbreviates "if and only if". Proofs are ended by the symbol $\square$. Sub-proofs inside one main proof are ended by the symbol $\dashv$.

We refer to [5] for standard set-theoretic notation. Let $X, Y$ be sets. $\mathscr{P}(X)$ denotes the set of all subsets of $X, X \times Y$ denotes the Cartesian product of $X$ and $Y, X-Y$ denotes the difference of $X$ and $Y,{ }^{X} Y$ denotes the set of all functions from $X$ to $Y$, ${ }^{[X]} Y$ denotes the set of all partial functions from $X$ to $Y$, namely functions from $Z$ to $Y$, where $Z$ is a subset of $X$. The domain of a function $f$ is denoted by $\operatorname{dom}(f)$, its range by $\operatorname{ran}(f)$. If $f$ is a function and $X \subseteq \operatorname{dom}(f), f\lceil X$ denotes the restriction of $f$ to $X$. Ordinal numbers are denoted by the initial letters of the Greek alphabet $\alpha, \beta, \gamma, \delta$. The set of all natural numbers is denoted by $\omega$ and the class of all ordinal numbers is denoted by On.

We refer to [10] for basic definitions and facts about partially ordered sets. Let $(P, \preceq)$ be a partially ordered set (a poset, for short). We say that two elements $p, p^{\prime}$ of $P$ are compatible (in $P$ ), writing $p \| p^{\prime}$, iff there exists an element $q \in P$ such that both $p \preceq q$ and $p^{\prime} \preceq q$ hold. Let $C$ be any subset of $P$. The least upper bound (or, the supremum) of $C$ in $P$ is denoted by $\operatorname{lub}_{P}(C)$. The maximum of $C$ is denoted by $\max (C)$. A poset $(P, \preceq)$ is a complete lattice iff for every subset $C$ of $P$ the least upper bound of $C$ in $P$ exists. A subset $C$ of $P$ is consistent (in $P$ ) iff every two elements of $C$ are compatible (in $P$ ). A poset $(P, \preceq$ ) is a coherent complete partial order (or, a cсpo) iff for every consistent subset $C$ of $P$ the least upper bound of $C$ in $P$ exists.

An operator $J: P \rightarrow P$ is monotone iff, for any $p, q \in P, p \preceq q \Rightarrow J(p) \preceq J(q)$. An element $p$ of $P$ is $J$-sound iff $p \preceq J(p)$, and is a fixed point of $J$ iff $p=J(p)$. The set of all fixed points of $J$ is denoted by $\operatorname{Fix}(J)$. If $(P, \preceq)$ is a ccpo, then for every $J$-sound element $p$ of $P$ there exists the least fixed point $p^{\prime}$ of $J$ above $p$, namely, $p \preceq p^{\prime}, p^{\prime} \in \operatorname{Fix}(J)$, and, for any other fixed point $q$ of $J$, if $p \subseteq q$ then $p^{\prime} \subseteq q$. We denote the least fixed point of $J$ above $p$ by $\operatorname{Ifp}(J, p)$. The least fixed point of $J$ above the bottom of $P$ is also the least fixed point of $J$, and will be denoted by $\operatorname{lfp}(J)$.

For each $J$-sound $p \in P$, define by transfinite recursion the following sequence of elements of $P:\left\langle J_{\alpha}^{p} \mid \alpha \in \mathrm{On}\right\rangle$, where $J_{0}^{p}=p, J_{\alpha+1}^{p}=J\left(J_{\alpha}^{p}\right), J_{\delta}^{p}=\operatorname{lub}_{P}\left\{J_{\alpha}^{p} \mid \alpha<\delta\right\}$ for $\delta$ limit. We call the sequence $\left\langle J_{\alpha}^{p} \mid \alpha \in \mathrm{On}\right\rangle$ the transfinite iteration of J starting with $p$. The sequence $\left\langle J_{\alpha} \mid \alpha \in \mathrm{On}\right\rangle$ will denote the transfinite iteration of $J$ starting with the bottom of $P$. It follows that $\operatorname{Ifp}(J)=J_{\alpha}$ for some $\alpha$ limit.

We will work in an abstract setting in which all mathematical objects will be considered relatively to a fixed, non-empty set $A$ (subsets of $A$, functions from $A$, etc.). We will reserve the following letters, variously decorated, to range over the corresponding classes of objects "from $A$ ":

1. $x, y, z$ for elements of $A$.

2. $X, Y, Z$ for subsets of $A$, i.e., elements of $\mathscr{P}(A)$.

3. $f, g, h$ for functions with domain $A$, i.e., elements of ${ }^{A} B$ for some set $B$. 
4. $p, q, r$ for partial functions on $A$, i.e., functions with domain a subset of $A$.

5. $R$ for binary relations on $A$, i.e., subsets of $A \times A$.

6. $\mathscr{X}$ for families of subsets of $A$.

7. $\mathscr{F}, \mathscr{G}$ for families of partial functions on $A$.

8. $\Delta$ for operators on $A$, i.e., functions from $\mathscr{P}(A)$ to $\mathscr{P}(A)$.

9. $\Gamma$ for operators $\Gamma: \mathscr{F} \rightarrow \mathscr{F}^{\prime}$ between families of partial functions on $A$.

We collect here few notions and lemmata on partially ordered sets and partial functions which will be useful in the subsequent sections.

\section{Order-theoretic intrinsicity}

Def. 1.2 Let $(P, \preceq)$ be a poset and let $C$ be a subset of $P$. We say that an element $p \in P$ is intrinsically in $C$ (or is $C$-intrinsic) iff $p$ belongs to $C$ and is compatible with any other element of $C$.

We denote the set of all $C$-intrinsic elements of $P$ by $\operatorname{lnt}(C)$, so

$$
\operatorname{lnt}(C)=\{p \in C \mid \forall q \in C(p \| q)\} .
$$

Definition 1.2 is an obvious generalisation of both (a) Kripke's notion of intrinsic fixed point [3, p. 709], which corresponds to taking $C$ to be the set of all fixed points of a monotone operator, and (b) Visser's notion of intrinsic element [10, p. 197], which corresponds to taking $C=P$.

Def. 1.3 Let $(P, \preceq)$ be a poset and let $C$ be a subset of $P$. We say that $C$ is intrinsically closed iff for every subset $C^{\prime} \subseteq \operatorname{lnt}(C)$, if $\operatorname{lub}_{P}\left(C^{\prime}\right)$ exists then $\operatorname{lub}_{P}\left(C^{\prime}\right) \in C$.

Lemma 1.4 Let $(P, \preceq)$ be a ccpo and let $C$ be a subset of $P$. If $C$ is intrinsically closed then, for every subset $C^{\prime} \subseteq \operatorname{Int}(C), \operatorname{lub}_{P}\left(C^{\prime}\right)$ exists and belongs to $\operatorname{lnt}(C)$.

Proof Let $C^{\prime} \subseteq \operatorname{Int}(C)$. By definition, $\operatorname{lnt}(C)$ is a consistent subset of $P$, hence $C^{\prime}$ is consistent too. Since $P$ is a ccpo, $\operatorname{lub}_{P}\left(C^{\prime}\right)$ exists. Since $C$ is intrinsically closed, $\operatorname{lub}_{P}\left(C^{\prime}\right) \in C$. We only have to show that $\operatorname{lub}_{P}\left(C^{\prime}\right)$ is compatible with every $p \in C$. Let $E=C^{\prime} \cup\{p\}$. The set $E$ is consistent in $P$, so $\operatorname{lub}_{P}(E)$ exists. Let $\bar{p}=\operatorname{lub}_{P}(E)$. For every $q \in C^{\prime}, q \preceq \bar{p}$, so $\operatorname{lub}_{P}\left(C^{\prime}\right) \preceq \bar{p}$. Since also $p \preceq \bar{p}$, $\operatorname{lub}_{P}\left(C^{\prime}\right)$ and $p$ are compatible.

Both the statement and the proof of Lemma 1.4 are straightforward generalisations of Visser [10, Theorem 20].

By Lemma 1.4, whenever $P$ is a ccpo and $C \subseteq P$ is intrinsically closed, then, for every $C^{\prime} \subseteq \operatorname{Int}(C)$ the least upper bound of $C^{\prime}$ in $(\operatorname{Int}(C), \preceq)$ exists. In particular, there exists the maximum of $\operatorname{lnt}(C)$.

Lemma 1.5 Let $P$ be a ccpo and let $J: P \rightarrow P$ be monotone. Then, a $J$-sound element $p \in P$ is intrinsically $J$-sound iff $\operatorname{Ifp}(J, p)$ is an intrinsic fixed point of $J$.

Proof Claim: Let $p$ be $J$-sound. Then $p$ is intrinsically $J$-sound $\operatorname{iff} \operatorname{Ifp}(J, p)$ is.

Proof of the claim. In one direction, let $p$ be intrinsically $J$-sound and let $p_{\alpha}$ denote the transfinite iteration of $J$ starting with $p_{0}=p$. We will show by induction that $p_{\alpha}$ is intrinsically $J$-sound for every $\alpha \in \mathrm{On}$. Suppose $p_{\alpha}$ is intrinsically $J$-sound and let $q$ be $J$-sound. Hence, there exists $r$ such that $p_{\alpha}, q \preceq r$. Since $J$ is monotone, $J\left(p_{\alpha}\right), J(q) \preceq J(r)$, so $J\left(p_{\alpha}\right), q \preceq J(r)$. Thus $J\left(p_{\alpha}\right)$ and $q$ are compatible. Since $J\left(p_{\alpha}\right)$ is $J$-sound, it is intrinsically $J$-sound. 
Let $\alpha$ be limit. By the inductive hypothesis, all $p_{\alpha}$ for $\alpha<\delta$ are intrinsically $J$-sound. Let $q$ be $J$-sound. Hence, the set $X=\left\{p_{\alpha} \mid \alpha<\delta\right\} \cup\{q\}$ is consistent. Let $\bar{q}=\operatorname{lub}_{P}(X)$. Hence $p_{\alpha} \preceq \bar{q}$ for every $\alpha<\delta$. Thus, $p_{\delta}=\operatorname{lub}_{P}\left\{p_{\alpha} \mid \alpha<\delta\right\} \preceq \bar{q}$, so $p_{\delta}$ and $q$ are compatible. Since $p_{\delta}$ is $J$-sound, it is intrinsically $J$-sound.

In the other direction, let $\operatorname{Ifp}(J, p)$ be intrinsically $J$-sound and let $q$ be $J$-sound. By the hypothesis, there exists $r$ such that $p \preceq \operatorname{Ifp}(J, p) \preceq r$ and $q \preceq r$. Hence $p$ is compatible with $q$, so $p$ is intrinsically $J$-sound.

We now turn to prove the Lemma.

In one direction, let $p$ be intrinsically $J$-sound and let $q \in \operatorname{Fix}(J)$. In particular, $q$ is $J$-sound. By the claim, Ifp $(J, p)$ is intrinsically $J$-sound, so it is compatible with $q$. Hence, $\operatorname{Ifp}(J, p)$ is an intrinsic fixed point of $J$.

In the other direction, let $p$ be $J$-sound and let $\operatorname{Ifp}(J, p)$ be an intrinsic fixed point of $J$. Let $q$ be $J$-sound. Let $\bar{q}=\operatorname{Ifp}(J, q)$. By the hypothesis, Ifp $(J, p)$ is compatible with $\bar{q}$, hence there exists $r$ such that $p \preceq \operatorname{Ifp}(J, p) \preceq r$ and $q \preceq \bar{q} \preceq r$. So $p$ and $q$ are compatible. Hence $p$ is intrinsically $J$-sound.

Determinacy Let $A, B$ be two non-empty sets. The set ${ }^{[A]} B$ of all partial functions from $A$ to $B$ partially ordered by inclusion is a ccpo.

Let $\mathscr{F} \subseteq{ }^{[A]} B$. We denote by $\operatorname{Dom}(\mathscr{F})$ the family of all domains of functions in $\mathscr{F}$, namely, $\operatorname{Dom}(\mathscr{F})=\{\operatorname{dom}(p) \mid p \in \mathscr{F}\}$. If $\mathscr{F}$ is a consistent subset of ${ }^{[A]} B$, then the map $p \mapsto \operatorname{dom}(p)$ is an order homomorphism between $\mathscr{F}$ and $\operatorname{Dom}(\mathscr{F})$ (both ordered by inclusion), namely, for every $p, q \in \mathscr{F}, p \subseteq q \Leftrightarrow \operatorname{dom}(p) \subseteq \operatorname{dom}(q)$. Whenever $X \in \operatorname{Dom}(\operatorname{Int}(\mathscr{F}))$ we denote by $\mathrm{p}_{X}$ the unique function $p$ in $\mathscr{F}$ such that $\operatorname{dom}(p)=X$.

Def. 1.6 Let $\Delta$ be a monotone operator on $A$, and let $\mathscr{F} \subseteq{ }^{[A]} B$. We say that $\Delta$ supports $\mathscr{F}$ iff for all $X, Y \subseteq A$,

1. $X \in \operatorname{Dom}(\operatorname{Int}(\mathscr{F}))$ implies $\Delta(X) \in \operatorname{Dom}(\operatorname{Int}(\mathscr{F}))$ and $X \subseteq \Delta(X)$.

2. $X \subseteq \Delta(X), Y \in \operatorname{Dom}(\operatorname{Int}(\mathscr{F}))$, and $X \subseteq Y$ implies $X \in \operatorname{Dom}(\operatorname{Int}(\mathscr{F}))$.

Lemma 1.7 Let $\Delta$ be a monotone operator on A, and let $\mathscr{F} \subseteq{ }^{[A]} B$. Suppose that $\mathscr{F}$ is intrinsically closed and that $\Delta$ supports $\mathscr{F}$. Let $p=\max (\operatorname{Int}(\mathscr{F}))$. Then,

1. $\operatorname{dom}(p) \in \operatorname{Fix}(\Delta)$, and

2. $p\lceil\operatorname{lfp}(\Delta) \in \operatorname{lnt}(\mathscr{F})$.

Proof $\quad$ Let $X=\operatorname{dom}(p)$. Since $X \in \operatorname{Dom}(\operatorname{Int}(\mathscr{F}))$ and $\Delta$ supports $\mathscr{F}, X \subseteq \Delta(X)$ and $\Delta(X) \in \operatorname{Dom}(\operatorname{Int}(\mathscr{F}))$. By the maximality of $p$ in $\operatorname{lnt}(\mathscr{F}), \Delta(X) \subseteq X$. Therefore $X \in \operatorname{Fix}(\Delta)$. Since $\Delta$ supports $\mathscr{F}, X \in \operatorname{Dom}(\operatorname{Int}(\mathscr{F}))$, Ifp $(\Delta) \subseteq \Delta(\operatorname{Ifp}(\Delta))$, and Ifp $(\Delta) \subseteq X$, it follows that $\operatorname{Ifp}(\Delta) \in \operatorname{Dom}(\operatorname{Int}(\mathscr{F}))$. Hence, there exists $q \in \operatorname{Int}(\mathscr{F})$ such that $\operatorname{Ifp}(\Delta)=\operatorname{dom}(q)$. Since $\operatorname{Ifp}(\Delta) \subseteq X=\operatorname{dom}(p), p\lceil\operatorname{Ifp}(\Delta)$ is well defined. Since $\operatorname{dom}(p\lceil\mid \mathrm{Ifp}(\Delta))=\operatorname{dom}(q)$, by intrinsicity, $p\lceil\operatorname{lfp}(\Delta)=q$, so $p\lceil\mid \operatorname{lfp}(\Delta) \in \operatorname{lnt}(\mathscr{F})$.

\section{A General Framework for Recursion}

In this section, Theorem 2.15, we provide a method of definition by recursion general enough to extend Montague's theorem in three directions: recursion by a valuation system, non-well-founded recursion, and recursion on a dependence operator. 
Montague's theorem Let $R \subseteq A \times A$ be any binary relation on the set $A$. For $x \in A$, we denote by $x^{R}$ the set of all $R$-predecessors of $x$ in $A$, namely, $x^{R}=\{y \in A \mid(y, x) \in R\}$.

Def. 2.1 Let $R \subseteq A \times A$ and $G: A \times{ }^{[A]} B \rightarrow B$. Let $p \in{ }^{[A]} B$ and $x \in \operatorname{dom}(p)$. We say that $p$ is $(G, R)$-recursive at $x$ iff

1. $x^{R} \subseteq \operatorname{dom}(p)$, and

2. $p(x)=G\left(x, p\left\lceil x^{R}\right)\right.$.

Further, we say that $p$ is $(G, R)$-recursive iff $p$ is $(G, R)$-recursive at $x$ for every $x \in \operatorname{dom}(p)$.

By using the terminology established in Definition 2.1, Montague's theorem can be reformulated as follows:

Fact 2.2 Let $R$ be a well-founded relation on a set $A$ and let $G: A \times{ }^{[A]} B \rightarrow B$ be any function. Then there exists one and only one $(G, R)$-recursive function $f$ from $A$ to $B$.

In the statement of Fact 2.2 we can identify two parameters and one explicit hypothesis. The two parameters are: (a) the function $G$, and (b) the binary relation $R$. The hypothesis is that $R$ is well-founded on the set $A$. Our goal is to provide a version of Montague's theorem that covers suitable generalisations of all three elements: $G$, $R$ and well-foundedness.

Let us start by making precise what we mean by "suitable generalisation", separately for each of the above-mentioned elements.

Valuation systems The first parameter in our formulation of Montague's theorem is a function $G$ defined on the Cartesian product $A \times{ }^{[A]} B$. However, in order to reconstruct a $(G, R)$-recursive function $p$ we only need to know the values $G$ takes at the pairs of the form $\left(x, p\left\lceil x^{R}\right)\right.$ for $x$ in the domain of $p$. This suggests that a possible kind of generalisation could allow $G$ to be only defined on some pairs of the Cartesian product $A \times{ }^{[A]} B$. This intuition can be better reformulated by exploiting a correspondence between such partial functions on $A \times{ }^{[A]} B$ and so-called "valuation systems".

A valuation system is a triple $\left(\mathscr{F}, \mathscr{F}^{\prime}, \Gamma\right)$, where both $\mathscr{F}$ and $\mathscr{F}^{\prime}$ are non-empty sets of functions and $\Gamma$ is an operator, $\Gamma: \mathscr{F} \rightarrow \mathscr{F}^{\prime}$.

By putting $A=\bigcup\left\{\operatorname{dom}(p) \mid p \in \mathscr{F} \cup \mathscr{F}^{\prime}\right\}$, and $B=\bigcup\left\{\operatorname{ran}(p) \mid p \in \mathscr{F} \cup \mathscr{F}^{\prime}\right\}$, we can think both $\mathscr{F}$ and $\mathscr{F}^{\prime}$ as sets of partial functions from $A$ to $B$. We will say that “ $\left(\mathscr{F}, \mathscr{F}^{\prime}, \Gamma\right)$ is a valuation system on $A^{\prime}$ " meaning that $A=\bigcup\left\{\operatorname{dom}(p) \mid p \in \mathscr{F} \cup \mathscr{F}^{\prime}\right\}$. Moreover, in the subsequent treatment we always assume that $\mathscr{F}^{\prime}=\operatorname{ran}(\Gamma)$ and, since $\mathscr{F}=\operatorname{dom}(\Gamma)$, we can identify a valuation system $\left(\mathscr{F}, \mathscr{F}^{\prime}, \Gamma\right)$ with the operator $\Gamma$ itself, thought as a partial operator on ${ }^{[A]} B$.

Given a function $G$ defined on all $A \times{ }^{[A]} B$ we can define a valuation system $\Gamma_{G}$ simply by putting

for every $x \in A$ and $p \in{ }^{[A]} B$.

$$
\Gamma_{G}(p)(x)=G(x, p)
$$

In the other direction, given a valuation system $\left(\mathscr{F}, \mathscr{F}^{\prime}, \Gamma\right)$, intuitively we can thing the expression $\mathrm{G}_{\Gamma}(x, p)$ as denoting "the value assigned by the system $\Gamma$ and the function $p$ to the argument $x$ ". Observe that, every time $x$ belongs to the domain of $\Gamma(q)$ for some $q \in \mathscr{F}$, we can use $\Gamma(q)$ to "evaluate" $x$, assigning to $x$ the value 
$\Gamma(q)(x)$. Hence, to say that $\Gamma$ and $p$ determine the value of $x$ can mean two distinct things: Either (a) we can use $\Gamma(p)$ to evaluate $x$, or (b) we can use $\Gamma(q)$ for some $q$ extending $p$, as long as the value $\Gamma(q)(x)$ does not depend on the choice of a specific $q$. Let us make precise this idea as follows.

Let $x \in A$ and $p \in{ }^{[A]} B$. We denote by $\mathrm{Q}_{\Gamma}(x, p)$ the set

$$
\mathrm{Q}_{\Gamma}(x, p)=\{q \in \mathscr{F} \mid p \subseteq q \wedge x \in \operatorname{dom}(\Gamma(q))\} .
$$

Observe that

1. $q \in \mathrm{Q}_{\Gamma}(x, p) \Rightarrow q \in \mathrm{Q}_{\Gamma}(x, q)$, and

2. $q \in \mathrm{Q}_{\Gamma}(x, p) \wedge p^{\prime} \subseteq p \Rightarrow q \in \mathrm{Q}_{\Gamma}\left(x, p^{\prime}\right)$.

Def. 2.3 Let $\left(\mathscr{F}, \mathscr{F}^{\prime}, \Gamma\right)$ be a valuation system and let $p \in{ }^{[A]} b$ and $x \in A$. We say that $x$ is $\Gamma$-determined by $p$ iff

$$
\mathrm{Q}_{\Gamma}(x, p) \neq \emptyset \wedge \forall q, q^{\prime} \in \mathrm{Q}_{\Gamma}(x, p)\left(\Gamma(q)(x)=\Gamma\left(q^{\prime}\right)(x)\right) .
$$

Def. 2.4 Let $\left(\mathscr{F}, \mathscr{F}^{\prime}, \Gamma\right)$ be a valuation system. Define a function $\mathrm{G}_{\Gamma}$ as follows:

$$
\operatorname{dom}\left(\mathrm{G}_{\Gamma}\right)=\left\{(x, p) \in A \times{ }^{[A]} B \mid p \in \mathrm{Q}_{\Gamma}(x, p) \vee x \text { is } \Gamma \text {-determined by } p\right\},
$$

and, for all $(x, p) \in \operatorname{dom}\left(\mathrm{G}_{\Gamma}\right)$,

$$
\mathrm{G}_{\Gamma}(x, p)=\left\{\begin{aligned}
\Gamma(p)(x) & \text { if } p \in \mathrm{Q}_{\Gamma}(x, p) \\
\Gamma(q)(x), \text { where } q \in \mathrm{Q}_{\Gamma}(x, p) & \text { otherwise. }
\end{aligned}\right.
$$

Clearly, the function $\mathrm{G}_{\Gamma}$ is well defined since, for every pair $(x, p)$ in its domain, if $p \notin \mathrm{Q}_{\Gamma}(x, p)$ then $x$ is $\Gamma$-determined by $p$, so the value of $\Gamma(q)(x)$ does not depend on the choice of $q \in \mathrm{Q}_{\Gamma}(x, p)$.

In general, the function $G_{\Gamma}$ associated to a valuation system $\Gamma$ by Definition 2.4 will be only partially defined on the Cartesian product $A \times{ }^{[A]} B$. Total functions $G: A \times{ }^{[A]} B \rightarrow B$ correspond to "complete" valuation systems, as established by the following Definition 2.5 and Lemma 2.6

Def. 2.5 We say that a valuation system $\left(\mathscr{F}, \mathscr{F}^{\prime}, \Gamma\right)$ is complete whenever

1. $\mathscr{F}={ }^{[A]} B$.

2. $\mathscr{F}^{\prime}={ }^{A} B$.

Lemma 2.6 There exists a one-to-one correspondence between functions of the form $G: A \times\left[{ }^{[A]} B \rightarrow B\right.$ and complete valuation systems $\left({ }^{[A]} B,{ }^{A} B, \Gamma\right)$ given by the following maps:

$\Gamma \mapsto \mathrm{G}_{\Gamma}$, and,

$G \mapsto \Gamma_{G}$.

Proof It is immediate to see that $\Gamma_{G}$ is complete for every $G$. Conversely, whenever $\Gamma$ is complete, for every $p \in{ }^{[A]} B$ and for every $x \in A, p \in \mathrm{Q}_{\Gamma}(x, p)$, hence $\mathrm{G}_{\Gamma}$ is a total function.

Let $G=\mathrm{G}_{\Gamma}$, and let $x \in A$ and $p \in{ }^{[A]} B$. Since $p \in \mathrm{Q}_{\Gamma}(x, p)$,

$$
\Gamma_{G}(x, p)=\mathrm{G}_{\Gamma}(x, p)=\Gamma(p)(x) .
$$

Hence, $\Gamma_{G}=\Gamma$.

Conversely, let $\Gamma=\Gamma_{G}$, and let $x \in A$ and $p \in{ }^{[A]} B$. Since $\Gamma_{G}$ is complete, $\mathrm{G}_{\Gamma}(x, p)=\Gamma_{G}(p)(x)=G(x, p)$. Hence $\mathrm{G}_{\Gamma}=G$. 
In the light of Lemma 2.6, we can identify total functions $G: A \times{ }^{[A]} B \rightarrow B$ with complete valuation systems on $A$ and try to generalise Montague's theorem by taking a generic valuation system $\Gamma$ to play the role of the parameter $G$ or, in other words, by allowing $G$ to be a partial function of the form $G=G_{\Gamma}$ for some valuation system $\Gamma$.

Non-well-foundedness Montague's theorem establishes existence and uniqueness of a $(G, R)$-recursive function $p$ defined on $A$ whenever $R$ is well-founded on $A$. We obtain an equivalent reformulation of Montague's theorem simply by dropping the requirement on the well-foundedness of $R$ on "all" $A$ and allowing $p$ to be defined only where $R$ is well-founded.

More precisely, following Aczel [1,p. 743] we say that $x \in A$ is $R$-well-founded ${ }^{2}$ iff there is no infinite descending $R$-chain of elements of $A$ starting with $x$, namely, iff there is no infinite sequence $\left\langle x_{n} \mid n \in \omega\right\rangle$ of elements of $X$ such that (a) $\left(x_{n+1}, x_{n}\right) \in R$ for every $n \in \omega$, and (b) $x_{0}=x$. The well-founded part of $R$, denoted by $\mathrm{W}(A, R)$, is the set of all $R$-well-founded elements of $A$.

Since a binary relation $R$ is well-founded on a set $A$ iff $A$ coincides with the wellfounded part of $R$, Fact 2.2 is equivalent to the following

Cor. 2.7 Let $R \subseteq A \times A$ and $G: A \times{ }^{[A]} B \rightarrow B$. There exists one and only one $(G, R)$-recursive function defined on the well-founded part of $R$.

Taken in the form given by Corollary 2.7, Montague's theorem establishes wellfoundedness as a criterion in order to get a subset $X$ of $A$ such that there exists one and only one $(G, R)$-recursive function defined on $X$. Well-foundedness is sufficient but not necessary in order to get the uniqueness of the $(G, R)$-recursive function (as we will see in the subsequent sections). This suggests the idea of extending Corollary 2.7 by replacing the well-founded part of $R$ with a subset of $A$ which is "as large as possible" as the domain of a unique $(G, R)$-recursive function. We will see that the abstract notion of intrinsicity can be applied to recursion to replace well-foundedness as a condition ensuring existence and uniqueness of such a "large" recursive function.

Dependence operators The second parameter in our formulation of Montague's theorem is a binary relation $R$ on $A$. Intuitively, $(G, R)$-recursiveness of $p$ at $x$ expresses the idea that the value of $p$ at $x$ "depends on" the restriction of $p$ to the set of all $R$-predecessors of $X$ or, in other words, that "to know the values of $p$ at each element of $x^{R}$ is sufficient in order to evaluate $p$ at $x$ ".

This intuition can be formalised by means of an operator $\Delta: \mathscr{P}(A) \rightarrow \mathscr{P}(A)$, reading " $x \in \Delta(X)$ " as " $x$ depends on $X$ ". The idea that " $X$ is sufficient for $x$ ", forces $\Delta$ to be monotone, namely, for all $X, Y \subseteq A, X \subseteq Y \Rightarrow \Delta(X) \subseteq \Delta(Y)$.

Given a binary relation $R \subseteq A \times A$, we can define a monotone operator $\Delta_{R}$ on $A$ by putting

for every $X \subseteq A$.

$$
\Delta_{R}(X)=\left\{x \in A \mid x^{R} \subseteq X\right\},
$$

In the other direction, given a monotone operator $\Delta$ on $A$, we would define a binary relation $R$ on $A$ such that, for every $x \in A, x \in \Delta\left(x^{R}\right)$. Let

$$
\mathrm{S}_{\Delta}(x)=\{X \subseteq A \mid x \in \Delta(X)\} .
$$

If, for every $x \in A$, there is a "natural" choice of an element $X_{x}$ in $\mathrm{S}_{\Delta}(x)$, we can simply define $R=\left\{(y, x) \in A \times A \mid y \in X_{x}\right\}$. However, such a "natural" choice is not 
always available. Binary relations can be identified with special kinds of monotone operators, called "essential-dependence operators" for which the "natural" choice is represented by the least element of $\mathrm{S}_{\Delta}(x)$. We recall here few things about essentialdependence operators, referring to Rivello [8] for proofs and further details.

Def. 2.8 Let $\Delta$ be a monotone operator on $A$. For every $x \in A$, define

$$
\mathrm{E}_{\Delta}(x)=\bigcap \mathrm{S}_{\Delta}(x)
$$

if $\mathrm{S}_{\Delta}(x) \neq \emptyset$; otherwise, $\mathrm{E}_{\Delta}(x)=A$.

$\Delta$ is an essential-dependence operator iff, for every $x \in A, \mathrm{E}_{\Delta}(x) \in \mathrm{S}_{\Delta}(x)$.

For a monotone operator $\Delta$ on $A$ to be an essential-dependence operator is equivalent to satisfy the following properties

1. (Surjectivity) For every $x \in A$ there exists $X \subseteq A$ such that $x \in \Delta(X)$.

2. (Generalised intersection property)

$$
\forall \mathscr{F}(\emptyset \neq \mathscr{F} \subseteq \mathscr{P}(A) \Rightarrow \bigcap\{\Delta(X) \mid X \in \mathscr{F}\} \subseteq \Delta(\bigcap \mathscr{F})) .
$$

Clearly, the Generalised intersection property implies the weaker Binary intersection property, namely,

$$
\forall X, Y \subseteq A(\Delta(X) \cap \Delta(Y) \subseteq \Delta(X \cap Y)) .
$$

Fact 2.9 The map $R \mapsto \Delta_{R}$ witnesses a one to one correspondence between binary relations on A and essential-dependence operators on A. Moreover, whenever $\Delta=\Delta_{R}$

$$
\mathrm{W}(A, R)=\operatorname{Ifp}(\Delta) .
$$

In the light of Fact 2.9, we can identify binary relations $R \subseteq A \times A$ with essentialdependence monotone operators on $A$ and try to generalise Montague's theorem by taking a generic monotone operator $\Delta$ to play the role of the parameter $R$, and by taking the least fixed point of $\Delta$ to play the role of the well-founded part of $R$.

Generalised recursion In the previous paragraphs we have argued that the notion of a valuation system $\Gamma$ on $A$ generalises that of a function $G$ defined on $A \times{ }^{[A]} B$, and that the notion of a monotone operator $\Delta$ on $A$ (and its least fixed point) generalises that of a binary relation $R$ on $A$ (and its well-founded part). Moreover, we have seen that we can generalise the statement of Corollary 2.7 also by asking for a $(G, R)$ recursive function uniquely defined on a "largest" subset of $A$ which extends the well-founded part of $R$.

In this paragraph we want to identify a notion of " $(\Gamma, \Delta)$-recursiveness" (and suitable conditions on $\Gamma$ and $\Delta$ ) such that we can prove a statement which simultaneously subsumes all three kinds of generalisations of Montague's theorem.

Given a monotone operator $\Delta$ on $A$, define

$$
\mathrm{C}_{\Delta}(x)=\left\{X \in \mathrm{S}_{\Delta}(x) \mid X \subseteq \mathrm{E}_{\Delta}(x)\right\} .
$$

Observe that

1. If there is no $X$ such that $x \in \Delta(X)$, then $C_{\Delta}(x)=\emptyset$.

2. If $\mathrm{E}_{\Delta}(x) \in \mathrm{S}_{\Delta}(x)$, then $\mathrm{C}_{\Delta}(x)=\left\{\mathrm{E}_{\Delta}(x)\right\}$.

3. If $\mathrm{S}_{\Delta}(x) \neq \emptyset$ but $\mathrm{E}_{\Delta}(x) \notin \mathrm{S}_{\Delta}(x)$, then $\mathrm{C}_{\Delta}(x)=\mathrm{S}_{\Delta}(x)$. 
Def. 2.10 Let $\left(\mathscr{F}, \mathscr{F}^{\prime}, \Gamma\right)$ be a valuation system on $A$ and let $\Delta$ be a monotone operator on $A$. Let $p \in{ }^{[A]} B$ and $x \in \operatorname{dom}(p)$. We say that $p$ is $(\Gamma, \Delta)$-recursive at $x$ iff

$$
\exists X \in \mathrm{C}_{\Delta}(x)\left(X \subseteq \operatorname { d o m } ( p ) \wedge \left(x, p\lceil X) \in \operatorname{dom}\left(\mathrm{G}_{\Gamma}\right) \wedge p(x)=\mathrm{G}_{\Gamma}(x, p\lceil X)) .\right.\right.
$$

Further, we say that $p$ is $(\Gamma, \Delta)$-recursive iff $p$ is $(\Gamma, \Delta)$-recursive at every $x$ in its domain.

When $\Gamma$ is complete, by Lemma 2.6, we can identify $\Gamma$ with a total function $G: A \times{ }^{[A]} B \rightarrow B$. When $\Delta$ is an essential-dependence operator, by Fact 2.9 , we can identify $\Delta$ with a binary relation $R \subseteq A \times A$. In the following Lemma 2.11 we check that, when $\Gamma$ is complete and $\Delta$ is an essential-dependence operator, we can equivalently use both definitions of recursiveness, either in terms of $(\Gamma, \Delta)$ or in terms of its corresponding pair $(G, R)$.

Lemma 2.11 Let $\Delta=\Delta_{R}$ and $\Gamma=\Gamma_{G}$, and let $x \in \operatorname{dom}(p)$. Then $p$ is $(\Gamma, \Delta)$ recursive at $x$ iff $p$ is $(G, R)$-recursive at $x$.

Proof In one direction, suppose $p$ is $(\Gamma, \Delta)$-recursive at $x$. Hence, there exists $X \in \mathrm{C}_{\Delta}(x)$ such that $X \subseteq \operatorname{dom}(p),\left(x, p\lceil X) \in \operatorname{dom}\left(\mathrm{G}_{\Gamma}\right)\right.$, and $p(x)=\mathrm{G}_{\Gamma}(x, p\lceil X)$.

By the hypothesis, $\mathrm{G}_{\Gamma}=G$ and $\Delta=\Delta_{R}$. Hence, $\mathrm{G}_{\Gamma}$ is total and $\mathrm{C}_{\Delta}(x)=\left\{x^{R}\right\}$. Therefore, $x^{R} \subseteq \operatorname{dom}(p)$ and $p(x)=G\left(x, p\left\lceil x^{R}\right)\right.$, namely, $p$ is $(G, R)$-recursive at $x$.

Conversely, suppose that $p$ is $(G, R)$-recursive at $x$. Hence, $x^{R} \subseteq \operatorname{dom}(p)$ and $p(x)=G\left(x, p\left\lceil x^{R}\right)\right.$. By the hypothesis, $G=\mathrm{G}_{\Gamma}$ and $\Delta=\Delta_{R}$. Let $X=x^{R}$. Hence, $X \in \mathrm{C}_{\Delta}(x), X \subseteq \operatorname{dom}(p),\left(x, p\lceil X) \in \operatorname{dom}\left(\mathrm{G}_{\Gamma}\right)\right.$, and $p(x)=\mathrm{G}_{\Gamma}(x, p\lceil X)$, namely, $p$ is $(\Gamma, \Delta)$-recursive at $x$.

Let $\operatorname{Rec}(\Gamma, \Delta)=\left\{p \in{ }^{[A]} B \mid p\right.$ is $(\Gamma, \Delta)$-recursive $\}$. The following Lemma 2.12 shows that we can use Lemma 1.4 in order to define the "largest" $(\Gamma, \Delta)$-recursive function for every choice of $\Gamma$ and $\Delta$.

Lemma 2.12 Let $\left(\mathscr{F}, \mathscr{F}^{\prime}, \Gamma\right)$ be a valuation system on $A$ and let $\Delta$ be a monotone operator on $A$. Then, $\operatorname{Rec}(\Gamma, \Delta)$ is intrinsically closed.

Proof Let $\mathscr{G} \subseteq \operatorname{lnt}(\operatorname{Rec}(\Gamma, \Delta))$ and let $p=\bigcup \mathscr{G}$. Let $x \in \operatorname{dom}(p)$. We have to show that $p$ is $(\Gamma, \Delta)$-recursive at $x$. Since $x \in \operatorname{dom}(p)$ there exists $q \in \mathscr{G}$ such that $x \in \operatorname{dom}(q)$. Since $q$ is $(\Gamma, \Delta)$-recursive,

$$
\exists X \in \mathrm{C}_{\Delta}(x)\left(X \subseteq \operatorname { d o m } ( q ) \wedge \left(x, q\lceil X) \in \operatorname{dom}\left(\mathrm{G}_{\Gamma}\right) \wedge q(x)=\mathrm{G}_{\Gamma}(x, q\lceil X)) .\right.\right.
$$

Since $q \subseteq p, X \subseteq \operatorname{dom}(p), p\lceil X=q\lceil X$ and $p(x)=q(x)$. Hence, $p$ is $(\Gamma, \Delta)$-recursive at $x$.

By Lemma 2.12 and Lemma 1.4 there exists the largest $(\Gamma, \Delta)$-recursive function which is compatible with any other $(\Gamma, \Delta)$-recursive function. We record this fact in the following

Def. 2.13 We denote by $\mathrm{F}(\Gamma, \Delta)$ the largest intrinsically $(\Gamma, \Delta)$-recursive function $p \in[A] B$, namely,

$$
\mathrm{F}(\Gamma, \Delta)=\max (\operatorname{Int}(\operatorname{Rec}(\Gamma, \Delta)))
$$

We denote by $F_{*}(\Gamma, \Delta)$ the union of all intrinsically $(\Gamma, \Delta)$-recursive functions whose domains are subsets of the least fixed point of $\Delta$. Clearly

$$
\mathrm{F}_{*}(\Gamma, \Delta)=\max \{p \in \operatorname{lnt}(\operatorname{Rec}(\Gamma, \Delta)) \mid \operatorname{dom}(p) \subseteq \operatorname{Ifp}(\Delta)\} .
$$


By definition, $F_{*}(\Gamma, \Delta) \subseteq F(\Gamma, \Delta)$. We call $F_{*}(\Gamma, \Delta)$ the grounded part of $F(\Gamma, \Delta)$.

Montague's theorem, in the formulation provided by Corollary 2.7, admits trivial instantiations. For instance, if $R$ is a reflexive binary relation, then $\mathrm{W}(A, R)=\emptyset$, and the theorem simply follows from the trivial fact that the empty function is $(G, R)$ recursive for any choice of $G$ (and $R$ ). The generalisation of Montague's theorem that follows from Lemma 2.12 can trivialise even when $\Delta$ corresponds to a binary relation $R$ which is well-founded on $A$. The reason is that Lemma 2.12 only grants the grounded part $F_{*}(\Gamma, \Delta)$ of $F(\Gamma, \Delta)$ to be well defined on a subset of the least fixed point of $\Delta$ : Nothing prevents the possibility that this subset could be very small, or even empty, for some choice of $\Gamma$ and $\Delta$.

This consideration leads us to restrict the scope of our generalisation of Montague's theorem only to those pairs $(\Gamma, \Delta)$ satisfying some conditions which ensure that the grounded part of $F(\Gamma, \Delta)$ is defined on all the least fixed point of $\Delta$, and, consequently, on all the well-founded part of $R$ in case $\Delta=\Delta_{R}$.

Def. 2.14 Let $\left(\mathscr{F}, \mathscr{F}^{\prime}, \Gamma\right)$ be a valuation system on $A$ and let $\Delta$ be a monotone operator on $A$. We say that the pair $(\Gamma, \Delta)$ admits recursion iff $\Delta$ supports $\operatorname{Rec}(\Gamma, \Delta)$.

We will see in the next Theorem 2.15 that if $(\Gamma, \Delta)$ admits recursion then $F_{*}(\Gamma, \Delta)$ is defined on all $\operatorname{lfp}(\Delta)$. Further, it turns out that admitting recursion is also a sufficient condition for another nice feature of $(\Gamma, \Delta)$-recursion. Let $p$ be $(\Gamma, \Delta)$-recursive at $x$. If $x$ does not depend essentially on $\mathrm{E}_{\Delta}(x)$, then $\mathrm{C}_{\Delta}(x)=\mathrm{S}_{\Delta}(x)$. This means that there exists at least one set $X$ in $\mathrm{S}_{\Delta}(x)$ witnessing that $p$ is $(\Gamma, \Delta)$-recursive at $x$, namely, that $p(x)=\mathrm{G}_{\Gamma}(x, p\lceil X)$ holds. However, in general, we do not have a criterion for picking a specific $X$ from $\mathrm{S}_{\Delta}(x)$. When $(\Gamma, \Delta)$ admits recursion and $p$ is intrinsically $(\Gamma, \Delta)$-recursive it happens that, to some extent, we do not have to make a choice since any $\Delta$-sound $X \in \mathrm{S}_{\Delta}(x)$ does the job.

Thm 2.15 (Generalised Montague's theorem) Suppose $(\Gamma, \Delta)$ admits recursion. Then there exists one and only one $(\Gamma, \Delta)$-recursive function $p$ defined on the least fixed point of $\Delta$. Moreover,

1. the domain of $\mathrm{F}(\Gamma, \Delta)$ is a fixed point of $\Delta$.

2. $p=\mathrm{F}_{*}(\Gamma, \Delta)=\mathrm{F}(\Gamma, \Delta)\lceil\operatorname{lfp}(\Delta)$.

3. Let $\bar{p}=\mathrm{F}(\Gamma, \Delta)$. For every $x \in \operatorname{dom}(\bar{p})$,

$$
\bar{p}(x)=\mathrm{G}_{\Gamma}(x, \bar{p}\lceil X),
$$

where $X$ is any set in $\mathrm{C}_{\Delta}(x)$ such that $(a) X \subseteq \Delta(X)$, (b) $X \subseteq \operatorname{dom}(\bar{p})$, and (c) $\left(x, \bar{p}\lceil X) \in \operatorname{dom}\left(\mathrm{G}_{\Gamma}\right)\right.$.

Proof By Lemma 2.12 and Lemma 1.7, the domain of $F(\Gamma, \Delta)$ is a fixed point of $\Delta$ and $\mathrm{F}_{*}(\Gamma, \Delta)=\mathrm{F}(\Gamma, \Delta)\lceil\operatorname{Ifp}(\Delta)$.

It only remains to show that, for every $x \in \operatorname{dom}(\bar{p}), \bar{p}(x)=\mathrm{G}_{\Gamma}(x, \bar{p} \mid X)$ for every $X \in \mathrm{C}_{\Delta}(x)$ such that $X \subseteq \operatorname{dom}(\bar{p})$ and $(x, \bar{p} \mid X) \in \operatorname{dom}\left(\mathrm{G}_{\Gamma}\right)$. Let $X$ be such a subset of $\operatorname{dom}(\bar{p})$. Since $X \subseteq \Delta(X)$ and $\Delta$ supports $\operatorname{Int}(\operatorname{Rec}(\Gamma, \Delta)), X \in \operatorname{Dom}(\operatorname{Int}(\operatorname{Rec}(\Gamma, \Delta)))$. Hence, there exists $q \in \operatorname{lnt}(\operatorname{Rec}(\Gamma, \Delta))$ such that $X=\operatorname{dom}(q)$. By intrinsicity, $q=\bar{p} \mid X$, namely, $\bar{p}\left\lceil X \in \operatorname{Int}(\operatorname{Rec}(\Gamma, \Delta))\right.$. Let $p^{\prime}=\bar{p}\left\lceil X \cup\left\{\left(x, \mathrm{G}_{\Gamma}(x, \bar{p}\lceil X))\right\}\right.\right.$. If $x \in X$ then $(\bar{p} \mid X)(x)=\bar{p}(x)=\mathrm{G}_{\Gamma}\left(x, \bar{p}\lceil X)\right.$, so $p^{\prime}$ is well defined. Let $y \in \operatorname{dom}\left(p^{\prime}\right)$. If $y \neq x$, then $y \in X$. Since $\bar{p}\left\lceil X\right.$ is $(\Gamma, \Delta)$-recursive at $y$, there exists $Y \in \mathrm{C}_{\Delta}(y)$ such that $Y \subseteq X=\operatorname{dom}\left(\bar{p}\lceil X),\left(y,\left(\bar{p}\lceil X)\lceil Y) \in \operatorname{dom}\left(\mathrm{G}_{\Gamma}\right)\right.\right.\right.$, and $\left(\bar{p}\lceil X)(y)=\mathrm{G}_{\Gamma}(y,(\bar{p}\lceil X)\lceil Y)\right.$. Since $Y \subseteq \operatorname{dom}\left(\bar{p}\lceil X)=X \subseteq \operatorname{dom}\left(p^{\prime}\right), p^{\prime}\left\lceil Y=\left(\bar{p}\lceil X)\left\lceil Y\right.\right.\right.\right.$, and $p^{\prime}(y)=\bar{p}\lceil X(y)$, 
$p^{\prime}$ is $(\Gamma, \Delta)$-recursive at $y$. If $y=x$, then $p^{\prime}$ is $(\Gamma, \Delta)$-recursive at $x$ by construction. Hence, $p^{\prime} \in \operatorname{Rec}(\Gamma, \Delta)$. By intrinsicity, $\bar{p}$ is compatible with $p^{\prime}$. Hence $\bar{p}(x)=p^{\prime}(x)=\mathrm{G}_{\Gamma}(x, \bar{p}\lceil X)$.

When $\Gamma=\Gamma_{G}$ and $\Delta=\Delta_{R}$ we already know from Montague's theorem that there exists a unique $(G, R)$-recursive function $p$ defined on the well-founded part of $R$ : By Lemma 2.11, $p$ is also the unique $(\Gamma, \Delta)$-recursive function defined on the least fixed point of $\Delta$. However, it can also be checked that $\left(\Gamma_{G}, \Delta_{R}\right)$ admits recursion for every choice of $G$ and $R$, so that, incidentally, Theorem 2.15 also provides an alternative proof of Montague's theorem. We will back on $(G, R)$-recursion in the subsequent sections, when we will study special cases of $(\Gamma, \Delta)$-recursion that correspond to different ways of generalising well-founded $(G, R)$-recursion and for which it is granted that $(\Gamma, \Delta)$ admits recursion.

We conclude this section proving a criterion that will prove useful in checking if a given pair $(\Gamma, \Delta)$ admits recursion.

Lemma 2.16 Let $\left(\mathscr{F}, \mathscr{F}^{\prime}, \Gamma\right)$ be any valuation system on $A$ and let $\Delta$ be a monotone operator on $A$. Let $\mathscr{X}=\operatorname{Dom}(\operatorname{Int}(\operatorname{Rec}(\Gamma, \Delta)))$ and suppose that

1. $\Delta$ has the "Binary intersection property", i.e., $\Delta(X) \cap \Delta(Y) \subseteq \Delta(X \cap Y)$,

2. $\mathscr{X}$ is " $\Delta$-closed", i.e., $X \in \mathscr{X}$ implies $\Delta(X) \in \mathscr{X}$, and

3. $\forall p \in \operatorname{Rec}(\Gamma, \Delta) \forall x \in \operatorname{dom}(p) \forall X \in \mathrm{C}_{\Delta}(x)$

$$
\left(X \subseteq \operatorname { d o m } ( p ) \Rightarrow \left(x, p\lceil X) \in \operatorname{dom}\left(\mathrm{G}_{\Gamma}\right) \wedge p(x)=\mathrm{G}_{\Gamma}(x, p\lceil X) .\right.\right.
$$

Then, $(\Gamma, \Delta)$ admits recursion.

Proof Let $\mathscr{G}=\operatorname{lnt}(\operatorname{Rec}(\Gamma, \Delta))$ and $\mathscr{X}=\operatorname{Dom}(\mathscr{G})$. By Definition 2.14 we have to show that $\Delta$ supports $\operatorname{Rec}(\Gamma, \Delta)$, namely, that for all $X, Y \subseteq A$,

1. $X \in \mathscr{X}$ implies $X \subseteq \Delta(X)$, and

2. $X \subseteq \Delta(X), Y \in \mathscr{X}$, and $X \subseteq Y$ implies $X \in \mathscr{X}$.

(1) Let $X \in \mathscr{X}$ and $x \in X$. Let $p \in \mathscr{G}$ be such that $\operatorname{dom}(p)=X$. Since $p$ is $(\Gamma, \Delta)$ recursive at $x$, there exists $Y \in C_{\Delta}(x)$ such that $Y \subseteq \operatorname{dom}(p)=X$. Since $x \in \Delta(Y)$ and $\Delta$ is monotone, $x \in \Delta(X)$. Thus, $X \subseteq \Delta(X)$.

(2) Let $X \subseteq \Delta(X), Y \in \mathscr{X}$, and $X \subseteq Y$. Let $p \in \mathscr{G}$ be such that $Y=\operatorname{dom}(p)$. Let $x \in X$. Since $x \in \operatorname{dom}(p)$, there exists $W \in \mathrm{C}_{\Delta}(x)$ such that $W \subseteq \operatorname{dom}(p)$ and $\left(x, p\lceil W) \in \operatorname{dom}\left(\mathrm{G}_{\Gamma}\right)\right.$. Let $Z=X \cap W$. Since $X \subseteq \Delta(X)$ and $W \in \mathrm{S}_{\Delta}(x)$, $x \in \Delta(X) \cap \Delta(W)$. Since $\Delta$ has the binary intersection property, $x \in \Delta(Z)$ and $Z \subseteq \Delta(Z)$. Since $\Delta$ is monotone, $\Delta(Z) \subseteq \Delta(W) \subseteq \mathrm{E}_{\Delta}(x)$, hence $Z \in \mathrm{C}_{\Delta}(x)$. By (2), $\left(x, p\lceil Z) \in \operatorname{dom}\left(\mathrm{G}_{\Gamma}\right)\right.$ and $p(x)=\mathrm{G}_{\Gamma}(x, p\lceil Z)$. Since $p\lceil Z=(p\lceil X)\lceil Z$, $\left(p\lceil X)(x)=p(x)=\mathrm{G}_{\Gamma}(x,(p\lceil X)\lceil Z)\right.$, hence $Z$ witnesses that $p\lceil X$ is $(\Gamma, \Delta)$ recursive at $x$. Since $p\lceil X$ is $(\Gamma, \Delta)$-recursive at $x$ for every $x \in X, p\lceil X \in \operatorname{Rec}(\Gamma, \Delta)$. Since $p$ is intrinsically $(\Gamma, \Delta)$-recursive, so is $p\lceil X$, hence $X \in \mathscr{X}$.

\section{Recursion on a Binary Relation}

In this section we study the special case of $(\Gamma, \Delta)$-recursion in which $\Delta=\Delta_{R}$, namely, we want to perform a definition by recursion along a binary relation $R \subseteq A \times A$.

Def. 3.1 Let $R$ be any binary relation on $A$ and let $\left(\mathscr{F}, \mathscr{F}^{\prime}, \Gamma\right)$ be any valuation system on $A$. Let $p \in{ }^{[A]} B$ and $x \in \operatorname{dom}(p)$. We say that $p$ is $(\Gamma, R)$-recursive at $x$ iff $p$ is $\left(\Gamma, \Delta_{R}\right)$-recursive at $x$. 
In the light of Definition 3.1, in this and in the subsequent section we write $\operatorname{Rec}(\Gamma, R), \mathrm{F}(\Gamma, R)$, and $\mathrm{F}_{*}(\Gamma, R)$ for $\operatorname{Rec}\left(\Gamma, \Delta_{R}\right), \mathrm{F}\left(\Gamma, \Delta_{R}\right)$, and $\mathrm{F}_{*}\left(\Gamma, \Delta_{R}\right)$, respectively.

Remark 3.2 Let $R$ be any binary relation on $A$ and let $\left(\mathscr{F}, \mathscr{F}^{\prime}, \Gamma\right)$ be any valuation system on $A$. Let $p \in{ }^{[A]} B$ and $x \in \operatorname{dom}(p)$. Then, $p$ is $(\Gamma, R)$-recursive at $x$ iff

$$
x^{R} \subseteq \operatorname{dom}(p) \wedge\left(x, p\left\lceil x^{R}\right) \in \operatorname{dom}\left(\mathrm{G}_{\Gamma}\right) \wedge p(x)=\mathrm{G}_{\Gamma}\left(x, p\left\lceil x^{R}\right) .\right.\right.
$$

In Theorem 3.5 below we will see that the function $\mathrm{F}(\Gamma, R)$ and its grounded part $\mathrm{F}_{*}(\Gamma, R)$ can always be characterised as the greatest and the least intrinsic fixed points (respectively) of a suitably defined monotone operator $J$ on ${ }^{[A]} B$.

Def. 3.3 Let $\left(\mathscr{F}, \mathscr{F}^{\prime}, \Gamma\right)$ be any valuation system on $A$ and let $R \subseteq A \times A$. Let $\mathrm{J}_{\Gamma, R}:{ }^{[A]} B \rightarrow{ }^{[A]} B$ denote the operator defined as follows. For every $p \in{ }^{[A]} B$,

1. $\operatorname{dom}\left(\mathrm{J}_{\Gamma, R}(p)\right)=\left\{x \in A \mid x^{R} \subseteq \operatorname{dom}(p) \wedge\left(x, p\left\lceil x^{R}\right) \in \operatorname{dom}\left(\mathrm{G}_{\Gamma}\right)\right\}\right.$, and

2. $\mathrm{J}_{\Gamma, R}(p)(x)=\mathrm{G}_{\Gamma}\left(x, p\left\lceil x^{R}\right)\right.$, for every $x \in \operatorname{dom}\left(\mathrm{J}_{\Gamma, R}(p)\right)$.

Observe that, for every $p \in{ }^{[A]} B, \operatorname{dom}\left(J_{\Gamma, R}(p)\right) \subseteq \Delta_{R}(\operatorname{dom}(p))$.

Lemma 3.4 $\mathrm{J}_{\Gamma, R}$ is monotone.

Proof Let $p \subseteq q$ and let $x \in \operatorname{dom}\left(\mathrm{J}_{\Gamma, R}\right)(p)$. By definition, $x^{R} \subseteq \operatorname{dom}(p)$ and $\left(x, p\left\lceil x^{R}\right) \in \operatorname{dom}\left(\mathrm{G}_{\Gamma}\right)\right.$. Since $q$ extends $p, x^{R} \subseteq \operatorname{dom}(q)$ and $p\left\lceil x^{R}=q\left\lceil x^{R}\right.\right.$. So, $x \in \operatorname{dom}\left(\mathrm{J}_{\Gamma, R}(q)\right)$. By definition,

$$
\mathrm{J}_{\Gamma, R}(q)(x)=\mathrm{G}_{\Gamma}\left(x, q\left\lceil x^{R}\right)=\mathrm{G}_{\Gamma}\left(x, p\left\lceil x^{R}\right)=\mathrm{J}_{\Gamma, R}(p) .\right.\right.
$$

Hence, $\mathrm{J}_{\Gamma, R}(p) \subseteq \mathrm{J}_{\Gamma, R}(q)$.

Thm 3.5 Let $R$ be any binary relation on $A$ and let $\left(\mathscr{F}, \mathscr{F}^{\prime}, \Gamma\right)$ be any valuation system on $A$. Then,

1. $\mathrm{F}(\Gamma, R)=\operatorname{gifp}\left(\mathrm{J}_{\Gamma, R}\right)$.

2. $\mathrm{F}_{*}(\Gamma, R)=\operatorname{lfp}\left(\mathrm{J}_{\Gamma, R}\right)$.

We will prove Theorem 3.5 after establishing a series of lemmata.

Lemma 3.6 $\quad p$ is $\mathrm{J}_{\Gamma, R}$-sound iff $p$ is $(\Gamma, R)$-recursive.

Proof $\quad$ Let $J=\mathrm{J}_{\Gamma, R}$. In one direction, suppose $p \subseteq J(p)$ and let $x \in \operatorname{dom}(p)$. Since $\operatorname{dom}(p) \subseteq \operatorname{dom}(J(p))$, it follows that $x^{R} \subseteq \operatorname{dom}(p)$ and $\left(x, p\left\lceil x^{R}\right) \in \operatorname{dom}\left(\mathrm{G}_{\Gamma}\right)\right.$. Since $p \subseteq J(p), p(x)=J(p)(x)=\mathrm{G}_{\Gamma}(x, p\lceil x)$, hence $p$ is $(\Gamma, R)$-recursive at $x$.

In the other direction, suppose $p$ is $\mathrm{J}_{\Gamma, R^{-}}$-sound and let $x \in \operatorname{dom}(p)$. Since $p$ is $(\Gamma, R)$-recursive at $x$, it follows that $x^{R} \subseteq \operatorname{dom}(p)$ and $\left(x, p\left\lceil x^{R}\right) \in \operatorname{dom}\left(\mathrm{G}_{\Gamma}\right)\right.$, so $x \in \operatorname{dom}(J(p))$. By definition of $J$,

$$
J(p)(x)=G\left(x, p\left\lceil x^{R}\right)=p(x) .\right.
$$

Hence $p \subseteq J(p)$.

Lemma 3.7 If $p$ is $(\Gamma, R)$-recursive so is $\mathrm{J}_{\Gamma, R}(p)$.

Proof Let $p(\Gamma, R)$-recursive. By Lemma 3.6, $p$ is $J_{\Gamma, R}$-sound. By Lemma 3.4, $\mathrm{J}_{\Gamma, R}$ is monotone, so $\mathrm{J}_{\Gamma, R}(p)$ is $\mathrm{J}_{\Gamma, R}$-sound too. Hence, by Lemma 3.6, $\mathrm{J}_{\Gamma, R}(p)$ is $(\Gamma, R)$-recursive.

Lemma 3.8 If $p$ is intrinsically $(\Gamma, R)$-recursive so is $\mathrm{J}_{\Gamma, R}(p)$. 
Proof Let $p \in \operatorname{lnt}(\operatorname{Rec}(\Gamma, R))$ and let $p^{\prime}=\mathrm{J}_{\Gamma, R}(p)$. By Lemma 3.7, $p^{\prime}$ is $(\Gamma, R)$ recursive. Let $q$ be $(\Gamma, R)$-recursive. Since $p$ is intrinsically $(\Gamma, R)$-recursive, there exists $r$ such that $p, q \subseteq r$. By Lemma 3.6, $q$ is $\mathrm{J}_{\Gamma, R^{-}}$sound and, by Lemma 3.4, $\mathrm{J}_{\Gamma, R}$ is monotone. Hence, $p^{\prime}=\mathrm{J}_{\Gamma, R}(p) \subseteq \mathrm{J}_{\Gamma, R}(r)$ and $q \subseteq \mathrm{J}_{\Gamma, R}(q) \subseteq \mathrm{J}_{\Gamma, R}(r)$. Therefore $p^{\prime}$ and $q$ are compatible, namely, $p^{\prime} \in \operatorname{Int}(\operatorname{Rec}(\Gamma, R))$.

Lemma 3.9 Let $p$ be a fixed point of $\rfloor_{\Gamma, R}$. Then $p$ is intrinsic iff $p$ is intrinsically $(\Gamma, R)$-recursive.

Proof Let $J=\mathrm{J}_{\Gamma, R}$. In one direction, let $p$ be an intrinsic fixed point of $J$. In particular, $\operatorname{Ifp}(J, p)=p$, hence, by Lemma $1.5, p$ is intrinsically $J$-sound. By Lemma $3.6, p$ is intrinsically $(\Gamma, R)$-recursive.

Conversely, let $p$ be intrinsically $(\Gamma, R)$-recursive. By Lemma 3.6, $p$ is intrinsically $J$-sound. Hence, by Lemma $1.5, \operatorname{Ifp}(J, p)=p$ is an intrinsic fixed point of $J$.

\section{Lemma 3.10 Let $p$ be $\mathrm{J}_{\Gamma, R}$-sound. Then}

1. $\operatorname{dom}(p)$ is $\Delta_{R}$-sound, and

2. $\operatorname{dom}\left(\operatorname{Ifp}\left(\mathrm{J}_{\Gamma, R}, p\right)\right) \subseteq \operatorname{Ifp}\left(\Delta_{R}, \operatorname{dom}(p)\right)$.

Proof Let $J=\mathrm{J}_{\Gamma, R}, \Delta=\Delta_{R}$, and let $p$ be $J$-sound.

(1) $\operatorname{dom}(p) \subseteq \operatorname{dom}(J(p)) \subseteq \Delta(\operatorname{dom}(p))$, so $\operatorname{dom}(p)$ is $\Delta$-sound. Define $p_{0}=p$, $p_{\alpha+1}=J(p)$, and $p_{\delta}=\bigcup\left\{p_{\alpha} \mid \alpha<\delta\right\}$ for $\delta$ limit, and define $X_{0}=\operatorname{dom}(p)$, $X_{\alpha+1}=\Delta\left(X_{\alpha}\right)$, and $X_{\delta}=\bigcup\left\{X_{\alpha} \mid \alpha<\delta\right\}$ for $\delta$ limit. We will show by transfinite induction that $\operatorname{dom}\left(p_{\alpha}\right) \subseteq X_{\alpha}$ for every $\alpha \in$ On. For $\alpha=0, \operatorname{dom}\left(p_{0}\right)=\operatorname{dom}(p)=X_{0}$. Let $\alpha=\beta+1$. Hence, by the inductive hypothesis and the monotonicity of $\Delta$,

$$
\operatorname{dom}\left(p_{\beta+1}\right)=\operatorname{dom}\left(J\left(p_{\beta}\right)\right) \subseteq \Delta\left(\operatorname{dom}\left(p_{\beta}\right)\right) \subseteq \Delta\left(X_{\beta}\right)=X_{\beta+1} .
$$

Let $\delta$ be limit and let $x \in \operatorname{dom}\left(p_{\delta}\right)$. By definition, $x \in \operatorname{dom}\left(p_{\alpha}\right)$ for some $\alpha<\delta$. By the inductive hypothesis, $\operatorname{dom}\left(p_{\alpha}\right) \subseteq X_{\alpha} \subseteq X_{\delta}$. Hence $x \in X_{\delta}$.

(2) Let $p_{\alpha}=\operatorname{Ifp}(J, p)$. By the construction of the least fixed point of $\Delta$ above $\operatorname{dom}(p), X_{\alpha} \subseteq \operatorname{Ifp}(\Delta, \operatorname{dom}(p))$, hence

$$
\operatorname{dom}(\operatorname{Ifp}(J, p))=\operatorname{dom}\left(p_{\alpha}\right) \subseteq X_{\alpha} \subseteq \operatorname{Ifp}(\Delta, \operatorname{dom}(p)) .
$$

Proof of Theorem 3.5 (1) $\mathrm{F}(\Gamma, R)=\operatorname{gifp}(J)$. Let $f=\mathrm{F}(\Gamma, R)$ and $g=\operatorname{gifp}(J)$. By Lemma 3.9, $g$ is intrinsically $(\Gamma, R)$-recursive, hence, by definition of $\mathrm{F}(\Gamma, R), g \subseteq f$. Conversely, by Lemma 3.6, $f$ is $J$-sound, hence there exists the least fixed point $p$ of $J$ above $f$. Since $f$ is intrinsically $J$-sound, by Lemma 1.5, $p$ is intrinsic. Hence, $f \subseteq p \subseteq g$.

(2) $\mathrm{F}_{*}(\Gamma, R)=\operatorname{Ifp}(J)$. Let $f_{*}=\mathrm{F}_{*}(\Gamma, R)$ and $g_{*}=\operatorname{Ifp}(J)$. By Lemma 3.9, $\operatorname{Ifp}(J)$ is intrinsically $(\Gamma, R)$-recursive and, by Lemma 3.10, its domain is a subset of $\operatorname{Ifp}\left(\Delta_{R}\right)$. Hence, $g_{*} \subseteq f_{*}$, by definition of $\mathrm{F}_{*}(\Gamma, R)$.

Conversely, since $\operatorname{dom}\left(f_{*}\right) \subseteq \operatorname{Ifp}\left(\Delta_{R}\right)=\mathrm{W}(A, R)$, we can show by $R$-induction that

$$
\forall x \in \operatorname{dom}\left(f_{*}\right)\left(x \in \operatorname{dom}\left(g_{*}\right) \wedge f_{*}(x)=g_{*}(x)\right),
$$

namely, that $f_{*} \subseteq g_{*}$. For, let $x \in \operatorname{dom}\left(f_{*}\right)$. Since $f_{*}$ is $(\Gamma, R)$-recursive, we know that $x^{R} \subseteq \operatorname{dom}\left(f_{*}\right)$ and that $\left(x, f\left\lceil x^{R}\right)\right.$ belongs to the domain of $\mathrm{G}_{\Gamma}$. By the inductive 
hypothesis, $x^{R} \subseteq \operatorname{dom}\left(g_{*}\right)$ and $f_{*}\left\lceil x^{R}=g_{*}\left\lceil x^{R}\right.\right.$. Hence $x \in \operatorname{dom}\left(J\left(g_{*}\right)\right)=\operatorname{dom}\left(g_{*}\right)$ and

$$
g_{*}(x)=J\left(g_{*}\right)(x)=\mathrm{G}_{\Gamma}\left(x, g_{*}\left\lceil x^{R}\right)=\mathrm{G}_{\Gamma}\left(x, f_{*}\left\lceil x^{R}\right)=f_{*}(x) .\right.\right.
$$

Actually, we did not use Lemma 3.8 in the proof of Theorem 3.5. However, Lemma 3.8 is useful in proving the following criterion for checking if a given pair $(\Gamma, R)$ admits recursion:

Lemma 3.11 Suppose that, for every $x \in A$ and $p \in \operatorname{lnt}(\operatorname{Rec}(\Gamma, R))$,

$$
x^{R} \subseteq \operatorname{dom}(p) \Rightarrow\left(x, p\left\lceil x^{R}\right) \in \operatorname{dom}\left(\mathrm{G}_{\Gamma}\right) .\right.
$$

Then, $(\Gamma, R)$ admits recursion.

Proof Let $\Delta=\Delta_{R}$. We prove the statement by showing that $\Delta$ satisfies all three hypotheses of Lemma 2.16 .

(1) Since $\Delta$ is an essential-dependence operator $\Delta$ has the Binary intersection property.

(2) Let $X \in \operatorname{Dom}(\operatorname{Int}(\operatorname{Rec}(\Gamma, \Delta)))$. By definition $\mathrm{p}_{X}$, the unique function in $\operatorname{Rec}(\Gamma, \Delta)$ whose domain is $X$, belongs to $\operatorname{Int}(\operatorname{Rec}(\Gamma, \Delta))$. Let $p=\mathrm{J}_{\Gamma, R}\left(\mathrm{p}_{X}\right)$. By Lemma 3.8, $p \in \operatorname{lnt}(\operatorname{Rec}(\Gamma, \Delta))$. As observed above, $\operatorname{dom}(p) \subseteq \Delta\left(\operatorname{dom}\left(\mathrm{p}_{X}\right)\right)=\Delta(X)$. Conversely, let $x \in \Delta\left(\operatorname{dom}\left(\mathrm{p}_{X}\right)\right)$. By definition, $x^{R} \subseteq \operatorname{dom}\left(\mathrm{p}_{X}\right)$, thus, by the hypothesis, $\left(x, \mathrm{p}_{X}\left\lceil x^{R}\right) \in \operatorname{dom}\left(\mathrm{G}_{\Gamma}\right)\right.$, namely, $x \in \operatorname{dom}(p)$. Therefore, $\Delta(X)=\operatorname{dom}(p)$, hence $\Delta(X) \in \operatorname{Dom}(\operatorname{Int}(\operatorname{Rec}(\Gamma, \Delta)))$.

(3) Let $p \in \operatorname{Rec}(\Gamma, \Delta), x \in \operatorname{dom}(p)$ and $X \in \mathrm{C}_{\Delta}(x)$. Since $p$ is $(\Gamma, \Delta)$-recursive at $x$ there exists $Y \in \mathrm{C}_{\Delta}(x)$ such that $Y \subseteq \operatorname{dom}(p),\left(x, p\lceil Y) \in \operatorname{dom}\left(\mathrm{G}_{\Gamma}\right)\right.$ and $p(x)=\mathrm{G}_{\Gamma}\left(x, p\lceil Y)\right.$. Since $\Delta$ is an essential-dependence operator, $X=x^{R}=Y$, so the last condition of Lemma 2.16 is satisfied.

Clearly, when $\Gamma$ is complete, namely, when $\Gamma=\Gamma_{G}$ for some total function $G$ on the domain $A \times{ }^{[A]} B$, the hypothesis of Lemma 3.11 is trivially satisfied. For an example of a pair $(\Gamma, R)$ admitting recursion even though $\Gamma$ is not complete, we can look at the Strong Kleene version of Kripke's theory of truth.

Let $A$ be the set of all sentences of $\mathscr{L}_{T r}$, where $\mathscr{L}_{T r}$ denotes the (first-order) language of arithmetic augmented by a unary predicate $\operatorname{Tr}$ (for "true"). Let $\kappa$ denote the Strong Kleene evaluation scheme, and let $\Gamma^{\mathcal{K}}$ denote the Kripkean monotone opera$\operatorname{tor}^{3}$ on ${ }^{[A]}\{\mathbf{t}, \mathbf{f}\}$ based on $\kappa$. Clearly, $\Gamma^{\kappa}$ is not complete: For instance, the sentence " $\operatorname{Tr}\ulcorner 0=0\urcorner$ " is not in the domain of $\Gamma^{\kappa}(\emptyset)$.

Let $\mathrm{R}$ denote the following binary relation defined on $A$. For sentences $\phi, \psi, \xi$, formula $\theta$ with $x$ the only free variable, and closed term $t$ :

$$
\begin{aligned}
\phi R \psi \leftrightarrow \quad & \psi=\neg \phi, \text { or } \\
& \psi=“ \phi \wedge \xi " \text { or } \psi=“ \xi \wedge \phi " \text { for some } \xi, \text { or } \\
& \psi=\forall x \theta(x) \text { and } \phi=\theta(t) \text { for some } \theta, t, \text { or } \\
& \psi=\operatorname{Tr}(\ulcorner\phi\urcorner) .
\end{aligned}
$$

Let $\mathrm{R}^{*}$ denote the transitive closure of $\mathrm{R}$. Then, we can prove, by induction on the syntax of $\mathscr{L}_{T r}$, that, for every $\phi \in A$ and $\left(\Gamma, \mathrm{R}^{*}\right)$-recursive $p \in{ }^{[A]} B$,

$$
\phi^{\mathrm{R}^{*}} \subseteq \operatorname{dom}(p) \Rightarrow \phi \in \operatorname{dom}\left(\Gamma\left(p\left\lceil\phi^{\mathrm{R}^{*}}\right)\right) .\right.
$$

Then, by Lemma 3.11, the pair $\left(\Gamma, R^{*}\right)$ admits recursion. 
Non-well-founded recursion Let us turn to revisit $(G, R)$-recursion in the light of the previously proved results. Clearly, taking $\Gamma=\Gamma_{G},(G, R)$-recursion is a special case of $(\Gamma, R)$-recursion. Moreover, since $G$ is total on $A \times{ }^{[A]} B$, the hypothesis of Lemma 3.11 is trivially satisfied, so $(G, R)$ admits recursion for any choice of $G$ and $R$. Hence, Theorem 2.15 provides an alternative proof of Montague's theorem, while Theorem 3.5 characterises the function $\mathrm{F}_{*}(G, R)$ defined by Montague's theorem and its extension $\mathrm{F}(G, R)$ as the least and the greatest fixed points, respectively, of the monotone operator $\mathrm{J}_{\Gamma, R}$, where $\Gamma=\Gamma_{G}$.

We write $\mathrm{J}_{G, R}$ for $\mathrm{J}_{\Gamma_{G}, R}$. The definition of $\mathrm{J}_{G, R}$ simplifies as follows: For every $p \in{ }^{[A]} B$,

1. $\operatorname{dom}\left(\mathrm{J}_{G, R}(p)\right)=\Delta_{R}(\operatorname{dom}(p))$, and

2. $\mathrm{J}_{G, R}(p)(x)=G\left(x, p\left\lceil x^{R}\right)\right.$, for every $x \in \operatorname{dom}\left(\mathrm{J}_{G, R}(p)\right)$.

Let us explicitly reformulate ${ }^{4}$ Theorem 2.15 for the specific case $\Gamma=\Gamma_{G}$ and $\Delta=\Delta_{R}$ :

Cor. 3.12 There exist both (a) the unique $(G, R)$-recursive function $p_{*}=\mathrm{F}_{*}(G, R)$ defined on the well-founded part of $R$, and $(b)$ the largest intrinsically $(G, R)$ recursive function $\bar{p}=\mathrm{F}(G, R)$. Moreover,

1. $\bar{p}$ is defined on a fixed point of the operator $\Delta_{R}$ associated to $R$, and

2. $p_{*}=\bar{p}\lceil\mathrm{~W}(A, R)$.

Theorem 2.15 really extends Montague's theorem when the domain of $\bar{p}$ properly extends the well-founded part of $R$. In this case we can appropriately call $\bar{p}=\mathrm{F}(G, R)$ "the function defined by non-well-founded recursion on the binary relation $R$ ".

For an example of a pair $(G, R)$ such that $\mathrm{F}(G, R)$ properly extends $\mathrm{F}_{*}(G, R)$ we can look at the Weak Kleene version of Kripke's theory of truth.

Let $\mathrm{R}$ denote the binary relation previously defined on the set $A$ of all sentences of the language $\mathscr{L}_{T r}$. Define $\mathrm{G}: A \times{ }^{[A]}\{\mathbf{t}, \mathbf{f}\} \rightarrow\{\mathbf{t}, \mathbf{f}\}$ by recursion on the syntax of $\mathscr{L}_{T r}$ as follows:

1. If $\psi=$ " $s=t$ ", then $\mathrm{G}(\psi, p)=\mathbf{t}$ iff $s^{\mathbb{N}}=t^{\mathbb{N}}$.

2. If $\psi=\neg \phi$, then $\mathrm{G}(\psi, p)=\mathbf{t}$ iff $p(\phi)=\mathbf{f}$.

3. If $\psi=\phi \wedge \xi$, then $\mathrm{G}(\psi, p)=\mathbf{t}$ iff $p(\phi)=p(\theta)=\mathbf{t}$.

4. If $\psi=\forall x \boldsymbol{\theta}(x)$, then $\mathrm{G}(\psi, p)=\mathbf{t}$ iff $p(\boldsymbol{\theta}(t))=\mathbf{t}$ for every closed term $t$.

5. If $\psi=\operatorname{Tr}\ulcorner\phi\urcorner$, then $\mathrm{G}(\psi, p)=\mathbf{t}$ iff $p(\phi)=\mathbf{t}$.

Let $\mu$ denote the Weak Kleene evaluation scheme, and let $\Gamma^{\mu}$ denote the Kripkean monotone operator ${ }^{5}$ on ${ }^{[A]}\{\mathbf{t}, \mathbf{f}\}$ based on $\mu$. Then, we can check, by arguing on the syntax of $\mathscr{L}_{T r}$, that

$$
\operatorname{Fix}\left(\Gamma^{\mu}\right)=\operatorname{Fix}\left(J_{G, R}\right) .
$$

It follows from Theorem 3.5 and Lemma 3.11, that

1. $F_{*}(G, R)=\operatorname{Ifp}\left(\Gamma^{\mu}\right)$, and

2. $F(G, R)=\operatorname{gifp}\left(\Gamma^{\mu}\right)$.

Since for the Kripkean operator $\Gamma^{\mu}$ the least fixed point and the greatest intrinsic fixed point are distinct, $F(G, R)$ properly extends $F_{*}(G, R)$.

\section{Recursion on the Dependence Operator}

We turn to another special case of $(\Gamma, \Delta)$-recursion. Its characteristic feature is that the dependence operator $\Delta$ is defined from the valuation system $\Gamma$ : 
Def. 4.1 Let $\left(\mathscr{F}, \mathscr{F}^{\prime}, \Gamma\right)$ be any valuation system on $A$. Define ${ }^{6}$

$$
\begin{aligned}
\Delta_{\Gamma}^{*}(X)=\left\{x \in A \mid \forall q, q^{\prime}\right. & \in \mathscr{F}\left(q \equiv_{X} q^{\prime} \wedge\right. \\
x & \left.\left.\in \operatorname{dom}(\Gamma(q)) \cap \operatorname{dom}\left(\Gamma\left(q^{\prime}\right)\right) \Rightarrow \Gamma(q)(x)=\Gamma\left(q^{\prime}\right)(x)\right)\right\},
\end{aligned}
$$

where $q \equiv_{X} q^{\prime}$ is short for " $X \subseteq \operatorname{dom}(q) \cap \operatorname{dom}\left(q^{\prime}\right) \wedge q \uparrow X=q^{\prime} \uparrow X$ ".

Lemma $4.2 \quad \Delta_{\Gamma}^{*}$ is monotone and surjective.

Proof (Monotonicity) Let $X \subseteq Y$ and $x \in \Delta_{\Gamma}^{*}(X)$. Let $q, q^{\prime} \in \mathscr{F}$ be such that $q \equiv_{Y} q^{\prime}$ and $x \in \operatorname{dom}(\Gamma(q)) \cap \operatorname{dom}\left(\Gamma\left(q^{\prime}\right)\right)$. Hence $q \equiv_{X} q^{\prime}$. So, $\Gamma(q)(x)=\Gamma\left(q^{\prime}\right)(x)$, namely, $x \in \Delta_{\Gamma}^{*}(Y)$.

(Surjectivity) Let $x \in A$. If there are no $q, q^{\prime} \in \mathscr{F}$ such that $q \equiv_{A} q^{\prime}$ and $x \in \operatorname{dom}(\Gamma(q)) \cap \operatorname{dom}\left(\Gamma\left(q^{\prime}\right)\right)$, then the implication holds vacuously. Otherwise, $q \equiv_{A} q^{\prime}$ implies $q=q^{\prime}$, hence $\Gamma(q)(x)=\Gamma\left(q^{\prime}\right)(x)$. Therefore, $x \in \Delta_{\Gamma}^{*}(A)$.

Remark 4.3 Observe that, for every $x \in A$ and $X \subseteq A, x \in \Delta_{\Gamma}^{*}(X)$ iff

$$
\forall p \in{ }^{X} B \forall q, q^{\prime} \in \mathrm{Q}_{\Gamma}(x, p)\left(\Gamma(q)(x)=\Gamma\left(q^{\prime}\right)(x)\right) .
$$

Hence, if $x \in \Delta_{\Gamma}^{*}(X)$ then, for every $p \in{ }^{X} B$, if $\mathrm{Q}_{\Gamma}(x, p) \neq \emptyset$ then $x$ is $\Gamma$-determined by $p$. Therefore, if $(x, p) \in \operatorname{dom}\left(\mathrm{G}_{\Gamma}\right), x \in \Delta_{\Gamma}^{*}(X)$ and $X \subseteq \operatorname{dom}(p)$, then

$$
\left(x, p\lceil X) \in \operatorname{dom}\left(\mathrm{G}_{\Gamma}\right) \wedge \mathrm{G}_{\Gamma}\left(x, p\lceil X)=\mathrm{G}_{\Gamma}(x, p) .\right.\right.
$$

By Lemma 4.2, the operator $\Delta_{\Gamma}^{*}$ can play the role of the dependence operator in a definition by $\left(\Gamma, \Delta_{\Gamma}^{*}\right)$-recursion for any valuation system $\Gamma$. In Theorem 4.5 below we give a sufficient condition on $\Gamma$ ensuring that the pair $\left(\Gamma, \Delta_{\Gamma}^{*}\right)$ admits recursion.

Def. 4.4 Let $\left(\mathscr{F}, \mathscr{F}^{\prime}, \Gamma\right)$ be any valuation system on $A$. We say that

1. $\Gamma$ is full iff ${ }^{A} B \subseteq \mathscr{F}$.

2. $\Gamma$ is regular iff for every $h \in{ }^{A} B \cap \mathscr{F}, \Gamma(h) \in{ }^{A} B$.

Observe that $\Gamma$ is both full and regular iff, for every $(x, p) \in A \times{ }^{[A]} B, \mathrm{Q}_{\Gamma}(x, p)$ is not empty. Hence, by Remark 4.3, if $x \in \Delta_{\Gamma}^{*}(X)$ then, for every $p$ such that $X \subseteq \operatorname{dom}(p), x$ is $\Gamma$-determined by $p$.

Thm 4.5 Let $\Gamma$ be monotone, full and regular. Then, $\left(\Gamma, \Delta_{\Gamma}^{*}\right)$ admits recursion.

Proof We prove the statement by showing that, whenever $\Gamma$ is monotone, full and regular, $\Delta_{\Gamma}^{*}$ satisfies all three hypotheses of Lemma 2.16. Let $\Delta=\Delta_{\Gamma}^{*}$.

Claim I: $\Delta$ has the binary intersection property.

Proof of Claim I. Let $x \in \Delta(X) \cap \Delta(Y)$ and let $q, q^{\prime} \in \mathscr{F}$ be such that $q \equiv_{X \cap Y} q^{\prime}$ and $x \in \operatorname{dom}(\Gamma(q)) \cap \operatorname{dom}\left(\Gamma\left(q^{\prime}\right)\right)$. Let $h, h^{\prime} \in{ }^{A} B$ be such that $q \subseteq h$ and $q^{\prime} \subseteq h^{\prime}$. Hence $h \equiv_{X \cap Y} h^{\prime}$. Since $\Gamma$ is full $h, h^{\prime} \in \mathscr{F}$. Since $\Gamma$ is monotone, $\Gamma(q) \subseteq \Gamma(h)$ and $\Gamma\left(q^{\prime}\right) \subseteq \Gamma\left(h^{\prime}\right)$. Let $g=h \uparrow X \cup h^{\prime} \uparrow(A / X)$. By construction, $g \equiv_{X} h$ and $g \equiv_{Y} h^{\prime}$ hold. Since $\Gamma$ is full, $g \in \mathscr{F}$. Since $\Gamma$ is regular, $x \in \operatorname{dom}(\Gamma)(g)$. Hence

$$
\Gamma(q)(x)=\Gamma(h)(x)=\Gamma(g)(x)=\Gamma\left(h^{\prime}\right)(x)=\Gamma\left(q^{\prime}\right)(x),
$$

namely, $x \in \Delta(X \cap Y)$.

Claim II: $\operatorname{Dom}(\operatorname{Int}(\operatorname{Rec}(\Gamma, \Delta))$ is $\Delta$-closed.

Proof of Claim II: Let $X \in \operatorname{Dom}(\operatorname{Int}(\operatorname{Rec}(\Gamma, \Delta)))$. Hence there exists (unique) $\mathrm{p}_{X} \in \operatorname{Int}(\operatorname{Rec}(\Gamma, \Delta))$ such that $\operatorname{dom}\left(\mathrm{p}_{X}\right)=X$. Since $\Gamma$ is full and regular, the set $\mathrm{Q}_{\Gamma}\left(x, \mathrm{p}_{X}\right)$ is not empty. By Remark 4.3, $x$ is $\Gamma$-determined by $\mathrm{p}_{X}$, hence, $\left(x, \mathrm{p}_{X}\right) \in \operatorname{dom}\left(\mathrm{G}_{\Gamma}\right)$. Therefore, we can define $p$ as follows 
1. $\operatorname{dom}(p)=\Delta(X)$, and

2. $p(x)=\mathrm{G}_{\Gamma}\left(x, \mathrm{p}_{X}\right)$, for every $x \in \operatorname{dom}(p)$.

Let $x \in X$. Since $\mathrm{p}_{X}$ is $(\Gamma, \Delta)$-recursive, $X \subseteq \Delta(X)=\operatorname{dom}(p)$ and there exists $Y \in \mathrm{C}_{\Delta}(x)$ such that $Y \subseteq X$ and $\mathrm{p}_{X}(x)=\mathrm{G}_{\Gamma}\left(x, \mathrm{p}_{X}\lceil Y)\right.$. By Remark 4.3, $x$ is $\Gamma$ determined by $\mathrm{p}_{X}\left\lceil Y\right.$, so $\mathrm{p}_{X}(x)=\mathrm{G}_{\Gamma}\left(x, \mathrm{p}_{X}\right)=p(x)$. Therefore, $\mathrm{p}_{X}=p\lceil X$. It follows that, by definition, for every $x \in \operatorname{dom}(p), p(x)=\mathrm{G}_{\Gamma}\left(x, \mathrm{p}_{X}\right)=\mathrm{G}_{\Gamma}(x, p\lceil X)$. If $\mathrm{E}_{\Delta}(x) \notin \mathrm{S}_{\Delta}(x)$, then $X \in \mathrm{C}_{\Delta}(x)$. Otherwise, $x \in \mathrm{E}_{\Delta}(x)$ and $\mathrm{E}_{\Delta}(x) \subseteq X=\operatorname{dom}\left(\mathrm{p}_{X}\right)$ so, by Remark 4.3, $p(x)=\mathrm{G}_{\Gamma}\left(x, p\left\lceil\mathrm{E}_{\Delta}(x)\right)\right.$. In both cases, there exists $Y \in \mathrm{C}_{\Delta}(x)$ witnessing that $p$ is $(\Gamma, \Delta)$-recursive at $x$, for every $x \in \operatorname{dom}(p)$. It remains to show that $p$ is intrinsic. Let $q$ be $(\Gamma, \Delta)$-recursive, and let $x \in \operatorname{dom}(p) \cap \operatorname{dom}(q)$. Since $q$ is $(\Gamma, \Delta)$-recursive at $x$, there exists $Y \in \mathrm{C}_{\Delta}(x)$ such that $Y \subseteq \operatorname{dom}(q),\left(x, q\lceil Y) \in \mathrm{G}_{\Gamma}\right.$, and $q(x)=\mathrm{G}_{\Gamma}(x, q \uparrow Y)$. Since $x \in \operatorname{dom}(p), x \in \Delta(X)$. Let $Z=X \cap Y$. By Claim I, $x \in \Delta(Z)$. By Remark 4.3, $p(x)=\mathrm{G}_{\Gamma}\left(x, p\lceil Z)\right.$ and $q(x)=\mathrm{G}_{\Gamma}(x, q\lceil Z)$. Since $Z \subseteq X$, $p\left\lceil Z=\mathrm{p}_{X}\left\lceil Z\right.\right.$. Since $\mathrm{p}_{X}$ is intrinsic, $\mathrm{p}_{X}\lceil Z=q\lceil Z$, hence $p(x)=q(x)$.

Claim III:

$$
\begin{aligned}
\forall p \in \operatorname{Rec}(\Gamma, \Delta) \forall x & \in \operatorname{dom}(p) \forall X \in \mathrm{C}_{\Delta}(x) \\
& \left(X \subseteq \operatorname { d o m } ( p ) \Rightarrow \left(x, p\lceil X) \in \operatorname{dom}\left(\mathrm{G}_{\Gamma}\right) \wedge p(x)=\mathrm{G}_{\Gamma}(x, p\lceil X)) .\right.\right.
\end{aligned}
$$

Proof of Claim III: Since $p$ is $(\Gamma, \Delta)$-recursive at $x$, there exists $Y \in \mathrm{C}_{\Delta}(x)$ such that $Y \subseteq \operatorname{dom}(p),\left(x, p\lceil Y) \in \mathrm{G}_{\Gamma}\right.$, and $p(x)=\mathrm{G}_{\Gamma}(x, p\lceil Y)$. Since $\Gamma$ is full and regular, $\mathrm{Q}_{\Gamma}(x, p)$ is not empty, hence, by Remark 4.3,

$$
p(x)=\mathrm{G}_{\Gamma}\left(x, p\lceil Y)=\mathrm{G}_{\Gamma}(x, p)=\mathrm{G}_{\Gamma}(x, p\lceil X) .\right.
$$

By Theorem 4.5, whenever $\Gamma$ is monotone, full and regular Theorem 2.15 applies. Moreover we can strengthen the third thesis of Theorem 2.15 to the following

Lemma 4.6 Let $\Gamma$ be monotone, full and regular. Let $\Delta=\Delta_{\Gamma}^{*}$. Let $\bar{p}=\mathrm{F}(\Gamma, \Delta)$. For every $x \in \operatorname{dom}(\bar{p})$,

$$
\bar{p}(x)=\mathrm{G}_{\Gamma}(x, \bar{p}\lceil X),
$$

where $X$ is any set in $\mathrm{S}_{\Delta}(x)$ such that $X \subseteq \operatorname{dom}(\bar{p})$.

Proof Let $x \in \operatorname{dom}(\bar{p})$. Since $\bar{p}$ is $(\Gamma, \Delta)$-recursive at $x$, there exists $Y \in \mathrm{C}_{\Delta}(x)$ such that $Y \subseteq \operatorname{dom}(\bar{p}),\left(x, \bar{p}\lceil Y) \in \operatorname{dom}\left(\mathrm{G}_{\Gamma}\right)\right.$, and $\bar{p}(x)=\mathrm{G}_{\Gamma}(x, \bar{p}\lceil Y)$.

Let $X \in \mathrm{S}_{\Delta}(x)$ be such that $X \subseteq \operatorname{dom}(p)$. Since $\Gamma$ is full and regular, there exists $h \in{ }^{A} B \subseteq \mathscr{F}$ such that $p \subseteq h$ and $x \in \operatorname{dom}(\Gamma(h))$. By Remark 4.3, $x$ is $\Gamma$-determined by both $p\left\lceil Y\right.$ and $p\left\lceil X\right.$. Hence $\left(x, p\lceil X) \in \operatorname{dom}\left(\mathrm{G}_{\Gamma}\right)\right.$ and

$$
\bar{p}(x)=\mathrm{G}_{\Gamma}\left(x, \bar{p}\lceil Y)=\Gamma(h)(x)=\mathrm{G}_{\Gamma}(x, \bar{p}\lceil X) .\right.
$$

We will see an example of definition by $\left(\Gamma, \Delta_{\Gamma}^{*}\right)$-recursion after some general consideration about the grounded part of $\mathrm{F}\left(\Gamma, \Delta_{\Gamma}^{*}\right)$. 
Grounded recursion Given a valuation system $\Gamma$ and a monotone operator $\Delta$ (on the same set $A$ ), Theorem 2.15 allows us to define a function $p$ on the least fixed point of $\Delta$ such that, for every $x$ in the domain of $p$, there exists a set $X \subseteq \operatorname{Ifp}(\Delta)$, depending on $x$, such that the value of $p$ at $x$ is "computable" from $p\lceil X$ by using the valuation system $\Gamma$.

My informal use of the term "computable" intends to suggest something more than "determinable": It wants to suggest that some "procedure" should be available in order to determine the value of $p$ at $x$. This should mean, at very least, that, for every $x$, (a) we have a way of choosing one set $X$, and (b) the elements of $X$ "come before" $x$ in some well ordering, so avoiding any kind of circularity or infinite regress.

This is indeed what happens when $\Delta$ is an essential-dependence operator, namely, an operator of the form $\Delta_{R}$ for a unique binary relation $R \subseteq A \times A$ : In this case, $\operatorname{Ifp}(\Delta)=\mathrm{W}(A, R)$, so (a) the subset $X$ is identified with the set $x^{R}$ of all predecessors of $x$ in $R$, and (b) the well-foundedness of $x$ in $R$ implies that the informal idea of "coming before" can formally be captured by the notion of " $R$-rank". For, recall that, for every $x \in \mathrm{W}(A, R)$, the $R$-rank of $x$, denoted by $\rho(x)$, is defined (by recursion on $R$ ) by $\rho(x)=\sup \left\{\rho(y)+1 \mid y \in x^{R}\right\}$. It follows that, for every $y \in x^{R}$, the $R$-rank of $y$ is strictly less than the $R$-rank of $x$ [5, Proposition 5.13, p. 67].

When $\Delta$ is not an essential-dependence operator, the informal idea of "coming before", associated to the "predecessors" of an element $x$ of Ifp $(\Delta)$, can formally be captured by the notion of " $\Delta$-rank". Let $\Delta$ be any monotone operator on $A$ and let $\left\langle\Delta_{\beta} \mid \beta \in \mathrm{On}\right\rangle$ denote the transfinite iteration of $\Delta$ starting with the empty set. For every $x \in \operatorname{Ifp}(\Delta)$ the $\Delta$-rank of $x$, denoted by $\alpha(x)$, is the least $\beta \in$ On such that $x \in \Delta_{\beta}$. Observe further that, if $\Delta=\Delta_{R}$, then, for every $x \in \operatorname{Ifp}(\Delta)=\mathrm{W}(A, R)$, $\alpha(x)=\rho(x)+1$.

The notion of $\Delta$-rank allows us to associate to each $x \in \operatorname{Ifp}(\Delta)$ the set $\Delta_{\alpha(x)-1}$, which is well defined since the $\Delta$-rank $\alpha(x)$ of $x$ is necessarily a successor ordinal.

By definition, $\Delta_{\alpha(x)-1}=\{y \in \operatorname{Ifp}(\Delta) \mid \alpha(y)<\alpha(x)\}$, so $\Delta_{\alpha(x)-1}$ can be thought as the set of all " $\Delta$-predecessors" of $x$.

When $\Delta$ is not an essential-dependence operator, Theorem 2.15 establishes that, in order to "compute" $p$ at $x$, we can choose any $Y \subseteq \operatorname{lfp}(\Delta)$ satisfying the following three conditions:

1. $x \in \Delta(Y)$,

2. $Y$ is $\Delta$-sound, and

3. $\left(x, p\lceil Y) \in \mathrm{G}_{\Gamma}\right.$.

Since, for every $\alpha, \Delta_{\alpha} \subseteq \Delta_{\alpha+1}$, and $\Delta_{\alpha+1}=\Delta\left(\Delta_{\alpha}\right)$, for every $x \in \operatorname{Ifp}(\Delta)$ the set $\Delta_{\alpha(x)-1}$ satisfies the first two conditions but, in general, the third condition is not granted.

When $\Gamma$ is monotone, full and regular, and $\Delta=\Delta_{\Gamma}^{*}$, Lemma 4.6 improves on Theorem 2.15 allowing us to choose any set $Y \subseteq \operatorname{lfp}(\Delta)$ which satisfies the first condition only: In particular, we can take $Y=\Delta_{\alpha(x)-1}$.

In other words, for every $x \in \operatorname{Ifp}(\Delta)$ we can make a canonical choice for the set $Y$ in $\mathrm{S}_{\Delta}(x)$ witnessing that $p_{*}=\mathrm{F}_{*}(\Gamma, \Delta)$ is $(\Gamma, \Delta)$-recursive at $x$.

Actually, to stick to our official definition of $(\Gamma, \Delta)$-recursiveness, we should pick the witness $Y$ from $\mathrm{C}_{\Delta}(x)$, and, whenever $x$ essentially depends on $\mathrm{E}_{\Delta}(x)$, the 
set $\Delta_{\alpha(x)-1}$ might not be in $\mathrm{C}_{\Delta}(x)$ : However, by Lemma 4.6, even in this case $p_{*}(x)=\mathrm{G}_{\Gamma}\left(x, p_{*}\left\lceil\mathrm{E}_{\Delta}(x)\right)=\mathrm{G}_{\Gamma}\left(x, p_{*}\left\lceil\Delta_{\alpha(x)-1}\right)\right.\right.$ holds.

A related consequence of Lemma 4.6 is that we can give an alternative definition of the grounded part of $\mathrm{F}(\Gamma, \Delta)$ arguing by transfinite recursion on the $\Delta$-rank, as established in the following

Lemma 4.7 Let $\Gamma$ be monotone, full and regular, and let $\Delta=\Delta_{\Gamma}^{*}$. Define by transfinite recursion on the $\Delta$-rank the following sequence $\left\langle p_{\beta} \mid \beta \in \mathrm{On}\right\rangle$ of binary relations on $A \times B$ :

1. $p_{0}=\emptyset$.

2. $p_{\beta+1}=p_{\beta} \cup\left\{\langle x, b\rangle \mid x \in \Delta_{\beta+1}-\Delta_{\beta} \wedge b=\mathrm{G}_{\Gamma}\left(x, p_{\beta}\right)\right\}$.

3. $p_{\delta}=\bigcup\left\{p_{\beta} \mid \beta<\delta\right\}$, for $\delta$ limit.

Let $p_{\infty}=\bigcup\left\{p_{\beta} \mid \beta \in\right.$ On $\}$. Then $p_{\infty}=\mathrm{F}_{*}(\Gamma, \Delta)$.

Proof First, observe that the sequence $\left\langle p_{\beta} \mid \beta \in \mathrm{On}\right\rangle$ is well defined. For, by transfinite induction we can check that $\operatorname{dom}\left(p_{\beta}\right)=\Delta_{\beta}$, for every $\beta \in \mathrm{On}$. Hence, in the successor clause of the definition, $x \in \Delta_{\beta+1}=\Delta\left(\Delta_{\beta}\right)$ and $\Delta_{\beta}=\operatorname{dom}\left(p_{\beta}\right)$ imply, by Remark 4.3, that $\left(x, p_{\beta}\right) \in \operatorname{dom}\left(\mathrm{G}_{\Gamma}\right)$.

Let $p_{*}=\mathrm{F}_{*}(\Gamma, \Delta)$. We will show by transfinite induction that, for every $\beta \in \mathrm{On}$,

$$
p_{\beta}=p_{*}\left\lceil\Delta_{\beta} .\right.
$$

If $\beta=0$, then $\Delta_{\beta}=\emptyset$. Hence, $p_{*}\left\lceil\Delta_{\beta}=\emptyset=p_{0}\right.$.

Let $\beta=\gamma+1$. By the inductive hypothesis, $p_{*}\left\lceil\Delta_{\gamma}=p_{\gamma}\right.$. Let $x \in \Delta_{\beta}-\Delta_{\gamma}$. By Lemma 4.6, $p_{*}(x)=\mathrm{G}_{\gamma}\left(x, p_{*}\left\lceil\Delta_{\gamma}\right)=\mathrm{G}_{\gamma}\left(x, p_{\gamma}\right)=p_{\beta}(x)\right.$. Hence, $p_{\beta}=p_{*}\left\lceil\Delta_{\beta}\right.$.

Let $\beta$ be limit. By the inductive hypothesis, $p_{*} \uparrow \Delta_{\gamma}=p_{\gamma}$ for every $\gamma<\delta$. Hence $p_{\beta}=\bigcup\left\{p_{\gamma} \mid \gamma<\beta\right\}=\bigcup\left\{p_{*}\left\lceil\Delta_{\gamma} \mid \gamma<\beta\right\}=p_{*}\left\lceil\Delta_{\beta}\right.\right.$.

For an application of the results illustrated in this section we can look at Leitgeb's definition of grounded truth. Leitgeb [4, p. 166] defines a monotone operator $D^{-1}$ on the set $A$ of all sentences of the language $\mathscr{L}_{T r}$. To every subset $Y \subseteq A$, Leitgeb associates a function $\operatorname{Val}_{Y}: A \rightarrow\{\mathbf{t}, \mathbf{f}\}$, and gives the following definition of dependence: For $y \in A$ and $X \subseteq A$, " $y$ depends on $X$ ", writing $y \in D^{-1}(X)$, iff for all $Y_{1}, Y_{2} \subseteq A$, if $Y_{1} \cap X=Y_{2} \cap X$ then $\operatorname{Val}_{Y_{1}}(y)=\operatorname{Val}_{Y_{2}}(y)$.

Every subset $Y \subseteq A$ can be identified with its characteristic function, namely with the function $\mathrm{h}_{Y}: A \rightarrow\{\mathbf{t}, \mathbf{f}\}$ such that $\mathrm{h}_{Y}(x)=\mathbf{t} \Leftrightarrow x \in Y$ holds for every $x \in A$. On the other hand, for every $Y \subseteq A, V a_{Y}$ itself is a characteristic function. Hence we can identify the map $Y \mapsto \mathrm{Val}_{Y}$ with an operator $\tau:{ }^{A}\{\mathbf{t}, \mathbf{f}\} \rightarrow{ }^{A}\{\mathbf{t}, \mathbf{f}\}$ on the set of all characteristic functions on $A$ into itself. Finally, if $h_{1}$ and $h_{2}$ are the characteristic functions of the subsets $Y_{1}, Y_{2}$, respectively, then $Y_{1} \cap X=Y_{2} \cap X$ holds iff $h_{1} \equiv_{X} h_{2}$ holds. Therefore, putting $\mathscr{F}=\mathscr{F}^{\prime}={ }^{A}\{\mathbf{t}, \mathbf{f}\}$ and $\Gamma=\tau$, Leitgeb's operator $D^{-1}$ turns out to be extensionally equivalent to the dependence operator $\Delta_{\Gamma}^{*}$.

For $\Gamma=\tau$, the valuation system $\left({ }^{A}\{\mathbf{t}, \mathbf{f}\},{ }^{A}\{\mathbf{t}, \mathbf{f}\}, \Gamma\right)$ is full and regular, by definition, and trivially monotone, since no two functions in ${ }^{A}\{\mathbf{t}, \mathbf{f}\}$ are comparable. It follows that Theorem 4.5 applies. Moreover, by Lemma 4.7, we can also characterise (the characteristic function of) Leitgeb's grounded truth $\Gamma_{l f}[4, \mathrm{p} .172]$, which was originally defined by transfinite recursion on the $\Delta_{\Gamma}^{*}$ rank, as the grounded part of the recursively defined function $\mathrm{F}\left(\Gamma, \Delta_{\Gamma}^{*}\right)$, namely, if we put $Y=\Gamma_{l f}$, then $\mathrm{h}_{Y}=\mathrm{F}_{*}\left(\Gamma, \Delta_{\Gamma}^{*}\right)^{7}$. 
Leitgeb's dependence operator also represents an interesting example in that, as showed by sentence 14 in Example list 1 [4, p. 164], for $\Gamma=\tau$, the operator $\Delta_{\Gamma}^{*}$ is not an essential-dependence operator, hence $\left(\Gamma, \Delta_{\Gamma}^{*}\right)$-recursion does not reduce to $(\Gamma, R)$-recursion.

\section{Notes}

1. See [5, p. 65]. The original formulation of the theorem given by Montague treats $G$ and $R$ as, possibly proper, classes. For the purposes of the present paper, we only need the set version of Montague's theorem which is given in Fact 1.1

2. Actually, this notion is called " $R$-groundedness" by many authors. We assume the axiom of Dependent Choice throughout the paper, so that $R$-groundedness is equivalent to the set-theoretic definition of $R$-well-foundedness. We rest on this latter terminology in order to avoid confusion with "groundedness with respect to a monotone operator", introduced immediately after Definition 2.13.

3. See Gupta and Belnap [2, pp. 41, 59] for definitions. In this book, our operator $\Gamma^{\kappa}$ corresponds to the operator $\kappa_{\mathbf{M}}$, taking $\mathbf{M}$ to be the standard model of arithmetic.

4. When $\Gamma=\Gamma_{G}$ and $\Delta=\Delta_{R}$, Corollary 3.12 can also be seen as a reformulation of the result already announced without proof in [7].

5. See Gupta and Belnap [2, pp. 41, 59] for definitions. In this book, our operator $\Gamma^{\mu}$ corresponds to the operator $\mu_{\mathbf{M}}$, taking $\mathbf{M}$ to be the standard model of arithmetic.

6. The star subscript in $\Delta_{\Gamma}^{*}$ is adopted in order to distinguish the operator $\Delta_{\Gamma}^{*}$ from the operator $\Delta_{\Gamma}$ introduced in [8]. In general, for every $X \subseteq A, \Delta_{\Gamma}(X) \subseteq \Delta_{\Gamma}^{*}(X)$.

7. Further details on this application of Theorem 2.15 to Leitgeb's theory of grounded truth are provided in [9, pp. 1249-1253].

\section{References}

[1] Aczel, P., “An introduction to inductive definitions,", in Handbook of Mathematical Logic, North Holland, 1977. 8

[2] Gupta, A., and N. Belnap, The Revision Theory of Truth, A Bradford Book. MIT Press, Cambridge, MA, 1993. 21

[3] Kripke, S., "Outline of a theory of truth," Journal of Philosophy, vol. 72 (1975), pp. 690716. 4

[4] Leitgeb, H., "What truth depends on,” Journal of Philosophical Logic, vol. 34 (2005), pp. 155-192. 20, 21

[5] Lévy, A., Basic Set Theory, Springer-Verlag, Berlin, 1979. 3, 19, 21

[6] Montague, R., "Well-founded relations: generalizations of principles of induction and recursion,” Bulletin American Mathematical Society, vol. 61 (1955), p. 442.1 
[7] Rivello, E., "On extending the general recursion theorem to non-wellfounded relations," in Logic Colloquium 2017, Abstracts of contributed talks, Bulletin of Symbolic Logic 24(2): pp. 262-263, 2018. 21, 22

[8] Rivello, E., "Formal representations of dependence and groundedness," The Review of Symbolic Logic, vol. 13 (2020), pp. 105-140. 9, 21

[9] Rivello, E., "Notes on Leitgeb's "What truth depends on"," Studia Logica, vol. 108 (2020), pp. 1235-1262. 21

[10] Visser, A., "Semantics and the Liar paradox,", in Handbook of Philosophical Logic, volume 4, Kluwer Academic Publishers, 1989. 3, 4

\title{
Acknowledgments
}

A substantially equivalent version of Corollary 3.12 was presented at the Logic Colloquium held in Stockholm in August 2017, and subsequently appeared as [7]. The other parts of the paper were partially presented at the Panhellenic Logic Symposium held in Anogeia (Crete) in June 2019 and at the Abstract objects and circularity workshop held in Muenchen in July 2019. I wish to thank the referees, the organisers and the audiences of these events and specially Thomas Schindler for inviting me in Muenchen and Catrin Campbell-Moore and Timo Beringer for their valuable comments. Finally, I want to thank the Department of Mathematics of the University of Torino and, in particular, Matteo Viale, for supporting me in covering the expenses to participate in the first event in 2017 (PRIN 2012, project n. 2012LZEBFL-003).

\author{
Department of Philosophy and Educational Sciences \\ University of Torino \\ 20 Via Sant'Ottavio \\ 10124 Torino \\ ITALY \\ rivello.edoardo@gmail.com
}

